\title{
Odd characteristic classes in entire cyclic homol- ogy and equivariant loop space homology
}

\author{
Sergio Cacciatori ${ }^{1}$ and Batu Güneysu ${ }^{2}$ \\ 1 Dipartimento di Scienza e Alta Tecnologia, Università dell'Insubria, via Valleggio 11, \\ 22100, Como, Italy and INFN - Sezione di Milano, via Celoria 16, 20133, Milano, Italy \\ email: sergio.cacciatori@uninsubria.it \\ ${ }^{2}$ Mathematisches Institut, Universität Bonn, Endenicher Allee 60, 53115 Bonn, Germany \\ email: gueneysu@math.uni-bonn.de
}

\begin{abstract}
Given a compact manifold $M$ and a smooth map $g: M \rightarrow U(l \times l ; \mathbb{C})$ from $M$ to the Lie group of unitary $l \times l$ matrices with entries in $\mathbb{C}$, we construct a Chern character $\mathrm{Ch}^{-}(g)$ which lives in the odd part of the equivariant (entire) cyclic Chen-normalized cyclic complex $\mathscr{N}_{\epsilon}\left(\Omega_{\mathbb{T}}(M \times \mathbb{T})\right)$ of $M$, and which is mapped to the odd Bismut-Chern character under the equivariant Chen integral map. It is also shown that the assignment $g \mapsto \mathrm{Ch}^{-}(g)$ induces a well-defined group homomorphism from the $K^{-1}$ theory of $M$ to the odd homology group of $\mathscr{N}_{\epsilon}\left(\Omega_{\mathbb{T}}(M \times \mathbb{T})\right)$.
\end{abstract}


Let $M$ be a closed Riemannian spin manifold with its Clifford multiplication

$$
c: \Omega(M) \longrightarrow \operatorname{End}(S)
$$

and its Dirac operator $D$ acting in $L^{2}(M, S)$, and given $g \in C^{\infty}(M, U(l \times l ; \mathbb{C}))$ let $D_{g}$ denote the twisted Dirac operator

$$
D_{g}:=g^{-1} D g=D+c\left(g^{-1} d g\right),
$$

considered to be acting on $L^{2}\left(M, S \otimes \mathbb{C}^{l}\right)$. Then with

$$
D_{g, s}:=(1-s) D+s D_{g}, \quad s \in[0,1],
$$

the odd dimensional variant of Atiyah-Singer's 'index' theorem states that if $M$ is odd dimensional, then 8 ]

$$
\frac{1}{2 \pi} \int_{0}^{1} \operatorname{Tr}\left[\dot{D}_{g, s} \exp \left(-D_{g, s}^{2}\right)\right] d s=\int_{M} \hat{A}(M) \wedge \operatorname{ch}^{-}(g),
$$

where $\mathrm{ch}^{-}(g) \in \Omega^{-}(M)$ denotes the odd Chern character. The left hand side of (11) is precisely the spectral flow $\operatorname{sf}\left(D, D_{g}\right)$ [ . Furthermore, on the RHS of this formula, the odd Chern character can be obtained integration along the fiber of $M \times I \rightarrow M$ of the even Chern character of an appropriately chosen connection on $M \times I$ [8]. In fact, this formula can be proved by noting the LHS admits an infinite dimensional version of such an even/odd periodicity [4, 5] in terms of the eta form.

Being motivated by the considerations of Atiyah and Bismut 1, 2, for the evendimensional case, one finds that another very elegant and geometric, however purely formal, way to prove (1) is to assume the existence of a DuistermaatHeckmann localization formula for the smooth loop space $L M$ : indeed, the spin structure on $M$ induces an orientation on $L M$ [1] and the path integral formalism entails the elegant, however mathematically ill-defined, formula (the evendimensional variant of this formula is well-known [2] and the odd-dimensional case can be proved similarly [14])

$$
\frac{1}{2 \pi} \int_{0}^{1} \operatorname{Tr}\left[\dot{D}_{g, s} \exp \left(-D_{g, s}^{2}\right)\right] d s=\int_{L M} \exp (-\beta) \wedge \operatorname{Bch}^{-}(g),
$$

where $\beta=\beta_{0}+\beta_{2} \in \Omega^{+}(L M)$ denotes the even differential form defined on smooth vector fields $X, Y$ on $L M$ by

$$
\beta_{0}(X):=\int_{0}^{1}\left|X_{s}\right|^{2} d s, \quad \beta_{2}(X, Y):=\int_{0}^{1}\left(\nabla X_{s} / \nabla s, Y_{s}\right) d s,
$$

and where $\mathrm{Bch}^{-}(g) \in \Omega^{-}(M)$ denotes the odd Bismut-Chern character [3, 18]. Now both differential forms $\exp (-\beta)$ and $\mathrm{Bch}^{-}(g)$ are equivariantly closed (cf. Section 4 for the definition of the degree -1 differential $P$ ),

$$
(d+P) \exp (-\beta)=0=(d+P) \operatorname{Bch}^{-}(g)
$$


and so is their product. As the fixed point set of the $\mathbb{T}$-action on $L M$ given by rotating every loop is precisely $M \subset L M$, a hypothetical Duistermaat-Heckmann localization formula immediately gives

$$
\int_{L M} \exp (-\beta) \wedge \mathrm{Bch}^{-}(g)=\left.\left.\int_{M} \hat{A}(M) \wedge \exp (-\beta)\right|_{M} \wedge \mathrm{Bch}^{-}(g)\right|_{M},
$$

as $\hat{A}(M)$ is the inverse of the (appropriately renormalized) Euler class of the normal bundle of $M \subset L M$. This proves (10), as clearly $\left.\exp (-\beta)\right|_{M}=1$ and by construction $\left.\mathrm{Bch}^{-}(g)\right|_{M}=\mathrm{ch}^{-}(g)$.

A direct implementation of the above arguments is not possible, as the right hand side of formula (2) is not well-defined for various reasons. For example, there exists no volume measure on $L M$, while smooth loops have Wiener measure zero, and, on the other hand, it is notoriously difficult to produce a variant of the super complex $(\Omega(L M), d+P)$ if one replaces $L M$ with the smooth Banach manifold of continuous loops. Nevertheless and strikingly, the above formal manipulations lead to the powerful machinery of hypoelliptic Dirac and Laplace operators, as is explained in [3] and the references therein.

However, a possible way out of these problems has been proposed by Getzler, Jones and Petrack (GJP) [1] 9]. In this approach, the idea is to take as model for $\Omega(L M)$ the space of equivariant Chen integrals: these are given by the image of a morphism of super complexes (cf. Section 4 below for the relevant definitions)

$$
\rho:\left(\mathscr{N}_{\epsilon}\left(\Omega_{\mathbb{T}}(M \times \mathbb{T})\right), b+B\right) \longrightarrow(\widehat{\Omega}(L M), d+P) .
$$

Above, $\mathscr{N}_{\epsilon}\left(\Omega_{\mathbb{T}}(M \times \mathbb{T})\right)$ denotes the Chen-normalized entire cyclic (or Connes) complex of the locally convex unital DGA $\Omega_{\mathbb{T}}(M \times \mathbb{T})$, and $\widehat{\Omega}(L M)$ denotes a completed space of smooth differential forms on $L M$. Now the GJP-program for infinite dimensional localization is as follows: here it is conjectured that the composition

$$
\int_{L M} \exp (-\beta) \wedge \rho(\cdot): \mathscr{N}_{\epsilon}\left(\Omega_{\mathbb{T}}(M \times \mathbb{T})\right) \longrightarrow \mathbb{C},
$$

is a mathematically well-defined continuous functional, and that

- $\int_{L M} \exp (-\beta) \wedge \rho(\cdot)$ is odd (as $L M$ is formally odd-dimensional if $M$ is so [3]) and co-closed, meaning that it vanishes on the exact elements of $\mathscr{N}_{\epsilon}\left(\Omega_{\mathbb{T}}(M \times\right.$ $\mathbb{T}))$,

- if $w \in \mathscr{N}_{\epsilon}\left(\Omega_{\mathbb{T}}(M \times \mathbb{T})\right)$ is closed, then one has the 'Duistermaat-Heckmann localization formula'

$$
\int_{L M} \exp (-\beta) \wedge \rho(w)=\left.\int_{M} \hat{A}(T M) \wedge \rho(w)\right|_{M} .
$$

If in addition one could canonically construct an element

$$
\mathrm{Ch}^{-}(g) \in \mathscr{N}_{\epsilon}^{-}\left(\Omega_{\mathbb{T}}(M \times \mathbb{T})\right)
$$

such that 
i) $\mathrm{Ch}^{-}(g)$ is closed

ii) $\rho\left(\mathrm{Ch}^{-}(g)\right)=\mathrm{Bch}^{-}(g)$

iii) $\left.\rho\left(\mathrm{Ch}^{-}(g)\right)\right|_{M}=\mathrm{ch}^{-}(g)$,

then from the above observations we would immediately obtain a proof of (1) within the GJP-program for infinite dimensional localization. Note that in the even dimensional case such a Chern character has been constructed as an even cycle in $\mathscr{N}_{\epsilon}\left(\Omega_{\mathbb{T}}(M \times \mathbb{T})\right)$ in [11].

The aim of this paper is precisely to construct a canonically given element

$$
\mathrm{Ch}^{-}(g) \in \mathscr{N}_{\epsilon}^{-}\left(\Omega_{\mathbb{T}}(M \times \mathbb{T})\right)
$$

satisfying the above properties i), ii), iii). In fact, our main results Theorem 5.1 and Theorem 5.4 below construct $\mathrm{Ch}^{-}(g)$ for $M$ a compact manifold (possibly with boundary), which satisfies i) and iii) and in addition ii) if $M$ is closed (so that $L M$ is a well-defined smooth Fréchet manifold). We also show in Theorem 5.1 that the assignment $g \mapsto \mathrm{Ch}^{-}(g)$ induces a well-defined group homomorphism

$$
\mathrm{K}^{-1}(M) \longrightarrow \mathscr{N}\left(\Omega_{\mathbb{T}}(M \times \mathbb{T})\right) .
$$

Finally, taking for granted that the even variant of $\mathrm{Ch}^{-}(g)$ and $\mathrm{BCh}^{-}(g)$ have been previously defined [11, 2, we establish an even/odd periodicity, relating these constructions to ours, showing another analogy to (1).

Acknowledgements: The authors would like to thank Jean-Michel Bismut, Markus Pflaum and Shu Shen for their discussions. We are very grateful to Matthias Ludewig for sharing his construction of the equivariant Chen integral map with us.

\section{Cyclic bar complex of a differential graded al- gebra (DGA)}

In the sequel, we understand all our linear spaces to be over $\mathbb{C}$. Assume we are given a unital DGA $\Omega$, that is,

- $\Omega$ is a unital algebra

- $\Omega=\bigoplus_{j=-\infty}^{\infty} \Omega^{j}$ is graded into subspaces $\Omega^{j} \subset \Omega$ such that $\Omega^{i} \Omega^{j} \subset \Omega^{i+j}$ for all $i, j \in \mathbb{Z}$, there is a degree +1 differential $d: \Omega \rightarrow \Omega$ which satisfies the graded Leibnitz rule.

Note that the space $\underline{\Omega}:=\Omega /(\mathbb{C} \cdot \mathbf{1})$ is a graded linear space (but not canonically an algebra), and the space of cyclic chains $\mathscr{C}(\Omega)$ is defined as

$$
\mathscr{C}(\Omega):=\bigoplus_{n=0}^{\infty} \Omega \otimes \underline{\Omega}^{\otimes n} .
$$


We give $\Omega \otimes \underline{\Omega}^{\otimes n}$ the grading

$$
\Omega \otimes \underline{\Omega}^{\otimes n}=\bigoplus_{j=0}^{\infty} \bigoplus_{j_{0}+\cdots+j_{n}=j-n} \Omega^{j_{0}} \otimes \underline{\Omega}^{j_{1}} \otimes \cdots \otimes \underline{\Omega}^{j_{n}},
$$

which induces a linear map

$$
\Gamma: \mathscr{C}(\Omega) \longrightarrow \mathscr{C}(\Omega), \quad \Gamma\left(w_{0}, w_{1}, \ldots\right):=\left((-1)^{\operatorname{deg}\left(w_{0}\right)} w_{0},(-1)^{\operatorname{deg}\left(w_{1}\right)} w_{1}, \ldots\right) .
$$

Since we have $\Gamma^{2}=1$, we can define a superstructure $\mathscr{C}(\Omega)=\mathscr{C}^{+}(\Omega) \oplus \mathscr{C}^{-}(\Omega)$ by setting

$$
\mathscr{C}^{ \pm}(\Omega):=\{w \in \mathscr{C}(\Omega): \Gamma w= \pm w\} .
$$

The following notation will be useful in the sequel:

Notation 1.1. Given $a \in \Omega \otimes \underline{\Omega}^{\otimes n}$ we define

$$
\langle a\rangle:=(\ldots, a, \ldots) \in \mathscr{C}(\Omega)
$$

to be the cochain which has $a$ in its $n$-th slot and 0 anywhere else.

We have the Hochschild map of the DGA-category

$$
b: \mathscr{C}(\Omega) \longrightarrow \mathscr{C}(\Omega)
$$

defined on $\Omega^{j_{0}} \otimes \underline{\Omega}^{j_{1}} \otimes \cdots \otimes \underline{\Omega}^{j_{n}}$ by

$$
\begin{aligned}
b\left\langle\omega_{0} \otimes \cdots \otimes \omega_{n}\right\rangle= & \left\langle d \omega_{0} \otimes \cdots \otimes \omega_{i} \otimes \cdots \otimes \omega_{n}\right\rangle \\
& -\sum_{i=1}^{n}(-1)^{j_{0}+\ldots+j_{i-1}-i+1}\left\langle\omega_{0} \otimes \cdots \otimes d \omega_{i} \otimes \cdots \otimes \omega_{n}\right\rangle \\
& -\sum_{i=0}^{n-1}(-1)^{j_{0}+\ldots+j_{i}-i}\left\langle\omega_{0} \otimes \cdots \otimes \omega_{i} \omega_{i+1} \otimes \cdots \otimes \omega_{n}\right\rangle \\
& +(-1)^{\left(j_{n}-1\right)\left(j_{0}+\ldots+j_{n-1}-n+1\right)}\left\langle\left(\omega_{n} \omega_{0}\right) \otimes \omega_{1} \otimes \cdots \otimes \omega_{n-1}\right\rangle,
\end{aligned}
$$

and Connes' operator

$$
B: \mathscr{C}(\Omega) \longrightarrow \mathscr{C}(\Omega)
$$

which is defined on $\Omega^{j_{0}} \otimes \underline{\Omega}^{j_{1}} \otimes \cdots \otimes \underline{\Omega}^{j_{n}}$ by

$B\left\langle\omega_{0} \otimes \cdots \otimes \omega_{n}\right\rangle=\sum_{i=0}^{n}(-1)^{\left(r_{i-1}+1\right)\left(r_{n}-r_{i-1}\right)}\left\langle 1 \otimes \omega_{i} \otimes \cdots \otimes \omega_{n} \otimes \omega_{0} \otimes \cdots \otimes \omega_{i-1}\right\rangle$, with $r_{l}=j_{0}+\cdots+j_{l}-l$. It is a well-known fact that one has

$$
b^{2}=0, \quad B^{2}=0, \quad b B+b B=0, \quad \Gamma b=-\Gamma b, \quad \Gamma B=-\Gamma B .
$$

We get the super complex

$$
\mathscr{C}^{+}(\Omega) \stackrel{b+B}{\longrightarrow} \mathscr{C}^{-}(\Omega) \stackrel{b+B}{\longrightarrow} \mathscr{C}^{+}(\Omega) .
$$


The subspace $\mathscr{D}(\Omega) \subset \mathscr{C}(\Omega)$ is defined to be the linear span of all $w \in \mathscr{C}(\Omega)$ that satisfy one of the following relations:

- for all $n \in \mathbb{N}$ there exists $1 \leq r \leq n, f \in \Omega^{0}, \omega_{0} \in \Omega, \omega_{s} \in \underline{\Omega}, s \neq r$, with

$$
\left\langle w_{n}\right\rangle=\left\langle\omega_{0} \otimes \cdots \otimes \omega_{r-1} \otimes f \otimes \omega_{r+1} \otimes \cdots \otimes \omega_{n}\right\rangle .
$$

- for all $n \in \mathbb{N}$ there exists $1 \leq r \leq n, f \in \Omega^{0}, \omega_{0} \in \Omega, \omega_{s} \in \underline{\Omega}, s \neq r$, with

$$
\begin{aligned}
& \left\langle\omega_{0} \otimes \cdots \otimes \omega_{r-1} f \otimes \omega_{r+1} \otimes \cdots \otimes \omega_{n}\right\rangle+\left\langle\omega_{0} \otimes \cdots \otimes \omega_{r-1} \otimes d f \otimes \omega_{r+1} \otimes \cdots \otimes \omega_{n}\right\rangle \\
& \quad-\left\langle\omega_{0} \otimes \cdots \otimes \omega_{r-1} \otimes f \omega_{r+1} \otimes \cdots \otimes \omega_{n}\right\rangle .
\end{aligned}
$$

The maps $\Gamma, b, B$ map $\mathscr{D}(\Omega)$ to itself, so that with

$$
\mathscr{D}^{ \pm}(\Omega):=\{w \in \mathscr{D}(\Omega): \Gamma w= \pm w\},
$$

there is a super complex

$$
\mathscr{D}^{+}(\Omega) \stackrel{b+B}{\longrightarrow} \mathscr{D}^{-}(\Omega) \stackrel{b+B}{\longrightarrow} \mathscr{D}^{+}(\Omega) .
$$

With $\mathscr{N}^{ \pm}(\Omega):=\mathscr{C}^{ \pm}(\Omega) / \mathscr{D}^{ \pm}(\Omega)$, the induced quotient complex

$$
\mathscr{N}^{+}(\Omega) \stackrel{b+B}{\longrightarrow} \mathscr{N}^{-}(\Omega) \stackrel{b+B}{\longrightarrow} \mathscr{N}^{+}(\Omega) .
$$

Whenever there is no danger of confusion, the equivalence class of $w \in \mathscr{C}(\Omega)$ in $\mathscr{N}(\Omega)$ is denoted with the same symbol again.

\section{Entire cyclic homology of a locally convex uni- tal DGA}

We recall that a topological vector space is called locally convex, if the topology is induced by a family of seminorms, noting that then the topology is equivalent to the topology induced by all continuous seminorms.

Definition 2.1. By a locally convex unital DGA we understand a unital DGA $\Omega$ which is also a locally convex Hausdorff space, such that

- the differential is continuous, e.g., for every continuous seminorm $\varepsilon$ on $\Omega$ there exists a continuous seminorm $\varepsilon^{\prime}$ on $\Omega$ such that

$$
\epsilon(d \omega) \leq \epsilon^{\prime}(\omega) \quad \text { for all } \omega \in \Omega
$$

- the multiplication is jointly continuous, e.g., for every continuous seminorm $\varepsilon$ on $\Omega$ there exists a continuous seminorm $\varepsilon^{\prime}$ on $\Omega$ such that

$$
\varepsilon\left(\omega_{1} \omega_{2}\right) \leq \varepsilon^{\prime}\left(\omega_{1}\right) \varepsilon^{\prime}\left(\omega_{2}\right) \quad \text { for all } \omega_{1}, \omega_{2} \in \Omega .
$$


The space $\underline{\Omega}$ becomes a graded locally convex Hausdorff space, and we equip the algebraic tensor product $\Omega \otimes \underline{\Omega}^{\otimes n}$ with the induced family of $\pi$-tensor seminorms, that is,

$$
\varepsilon_{n}(\omega)=\inf \left\{\sum_{\alpha} \varepsilon\left(\omega_{0}^{(1)}\right) \cdots \varepsilon\left(\omega_{n}^{(\alpha)}\right): \omega=\sum_{\alpha} \omega_{0}^{(\alpha)} \otimes \cdots \otimes \omega_{n}^{(\alpha)}\right\},
$$

where the sum runs through all representations of $\omega$ as a finite sum of elementary tensors, and where $\epsilon$ is a continuous seminorm on $\Omega$.

Definition 2.2. The space of entire cyclic chains $\mathscr{C}_{\epsilon}(\Omega)$ is defined to be the closure of $\mathscr{C}(\Omega)$ with respect to the seminorms

$$
\kappa_{\varepsilon}(w):=\sum_{n=0}^{\infty} \frac{\varepsilon_{n}\left(w_{n}\right)}{\sqrt{n !}},
$$

where $\varepsilon$ is an arbitrary continuous seminorm on $\Omega$.

The space $\mathscr{C}_{\epsilon}(\Omega)$ is a complete locally convex Hausdorff space. Note that the above family of seminorms is equivalent to the familiy of seminorms

$$
\kappa_{\varepsilon, l}(w):=\sum_{n=0}^{\infty} \frac{\varepsilon_{n}\left(w_{n}\right) l^{n}}{\sqrt{n !}}<\infty,
$$

where $\varepsilon$ is an arbitrary continuous seminorm on $\Omega$ and $l \in \mathbb{N}$, as $l \varepsilon$ is again a continuous seminorm and the $\varepsilon_{n}$ 's are cross semi-norms. Thus, our growth conditions are modelled on the entire growth conditions for ungraded Banach algebras by Getzler/Szenes from [12.

Before stating the next auxiliary result, we recall that a continous linar map from a locally convex Hausdorff space $\mathscr{X}$ to a complete locally convex Hausdorff space $\mathscr{Y}$ can be uniquely extended to a continuous linear map $\hat{\mathscr{X}} \rightarrow \mathscr{Y}$, noting that the completion $\hat{\mathscr{X}}$ is Hausdorff again. This can be proved precisely as for normed spaces.

Lemma 2.3. The operators $\Gamma, b, B$ map $\mathscr{C}(\Omega)$ continuously to itself, in particular, with

$$
\mathscr{C}_{\epsilon}^{ \pm}(\Omega):=\left\{w \in \mathscr{C}_{\epsilon}(\Omega): \Gamma w= \pm w\right\},
$$

there is a well-defined super complex

$$
\mathscr{C}_{\epsilon}^{+}(\Omega) \stackrel{b+B}{\longrightarrow} \mathscr{C}_{\epsilon}^{-}(\Omega) \stackrel{b+B}{\longrightarrow} \mathscr{C}_{\epsilon}^{+}(\Omega) .
$$

Proof. Let $\varepsilon$ be an arbitrary continuous seminorm on $\Omega$. Clearly, one has $\kappa_{\varepsilon}(\Gamma w) \leq$ $\kappa_{\varepsilon}(w)$ for all $w \in \mathscr{C}(\Omega)$.

Pick continuous seminorms $\varepsilon^{\prime}, \varepsilon^{\prime \prime}$ on $\Omega$ such that for all $\omega \in \Omega$ one has $\varepsilon(d \omega) \leq$ $\varepsilon^{\prime \prime}(\omega)$ and such that for all $\omega_{1}, \omega_{2} \in \Omega$ one has $\varepsilon\left(\omega_{1} \omega_{2}\right) \leq \varepsilon^{\prime}\left(\omega_{1}\right) \varepsilon^{\prime}\left(\omega_{2}\right)$. Using $n+1 \leq 2^{n}$ it is then easily checked that

$$
\kappa_{\varepsilon}(b w) \leq C \max \left(\kappa_{\varepsilon^{\prime}}, \kappa_{\varepsilon^{\prime \prime}}\right)(w) \quad \text { for all } w \in \mathscr{C}(\Omega) .
$$

Likewise, it follows immediately that $\kappa_{\varepsilon}(B w) \leq C \kappa_{\varepsilon}(w)$ for all $w \in \mathscr{C}(\Omega)$. 
Defining the subspace $\mathscr{D}_{\epsilon}(\Omega) \subset \mathscr{C}_{\epsilon}(\Omega)$ as the closure of $\mathscr{D}(\Omega)$, it follows automatically that the maps $\Gamma, b, B$ map $\mathscr{D}(\Omega)$ continuously to itself, too, producing with

$$
\mathscr{N}_{\epsilon}^{ \pm}(\Omega):=\mathscr{C}_{\epsilon}^{ \pm}(\Omega) / \mathscr{D}_{\epsilon}^{ \pm}(\Omega)
$$

the quotient complex

$$
\mathscr{N}_{\epsilon}^{+}(\Omega) \stackrel{b+B}{\longrightarrow} \mathscr{N}_{\epsilon}^{-}(\Omega) \stackrel{b+B}{\longrightarrow} \mathscr{N}_{\epsilon}^{+}(\Omega) .
$$

Finally we can give:

Definition 2.4. The complex (8) is called the (reduced) entire cyclic complex of $\Omega$ and its homology groups are denoted with $\operatorname{HC}_{\epsilon}^{ \pm}(\Omega)$. Likewise, the complex (9) is called the (reduced) Chen-normalized entire cyclic complex of $\Omega$ and its homology groups are denoted with $\operatorname{HN}_{\epsilon}^{ \pm}(\Omega)$.

Above, 'reduced' refers to the fact that we work with $\Omega \otimes \underline{\Omega}^{\otimes n}$ rather than $\Omega^{\otimes(n+1)}$, which leads to a simpler formula for the Connes differential $B$.

\section{$3 \quad$ The unital locally convex DGA $\Omega_{\mathbb{T}}(N \times \mathbb{T})$}

Assume $N$ is a manifold (possibly with boundary) and denote with $\mathbb{T}$ the 1 -sphere. We denote by $\Omega_{\mathbb{T}}(N \times \mathbb{T})$ the smooth $\mathbb{T}$-invariant differential forms on $N \times \mathbb{T}$, where $\mathbb{T}$ acts trivially on $N$ and by rotation on itself. Every element of $\Omega_{\mathbb{T}}(N \times \mathbb{T})$ can be uniquely written in the form $\alpha+\vartheta_{\mathbb{T}} \wedge \beta$ for some $\alpha, \beta \in \Omega(N)$, where $\vartheta_{\mathbb{T}}$ denotes the canonical 1-form on $\mathbb{T}$. We turn $\Omega_{\mathbb{T}}(N \times \mathbb{T})$ into a unital algebra by means of $\Omega_{\mathbb{T}}(N \times \mathbb{T}) \subset \Omega(N \times \mathbb{T})$, and give $\Omega_{\mathbb{T}}(N \times \mathbb{T})$ the grading

$$
\alpha+\vartheta_{\mathbb{T}} \wedge \beta \in \Omega_{\mathbb{T}}^{j}(N \times \mathbb{T}) \quad \Longleftrightarrow \alpha \in \Omega^{j}(N), \beta \in \Omega^{j+1}(N) .
$$

With $\partial_{\mathbb{T}}$ the canonical vector field on $\mathbb{T}$, we have the differential $d_{\mathbb{T}}=d+\iota_{\partial_{\mathbb{T}}}$ defined by

$$
d_{\mathbb{T}}\left(\alpha+\vartheta_{\mathbb{T}} \wedge \beta\right)=d \alpha+\beta-\vartheta_{\mathbb{T}} \wedge d \beta, \quad \text { if } \alpha+\vartheta_{\mathbb{T}} \wedge \beta \text { is homogeneous, }
$$

finally turning $\Omega_{\mathbb{T}}(N \times \mathbb{T})$ into a unital DGA.

Remark 3.1. Given a manifold $X$ (possibly with boundary), the wedge product and the de Rham differential is continuous with respect to the canonical locally convex structure on $\Omega(X)$ [17. In addition, if $B$ is a vector field on $X$ then the contraction

$$
\iota_{B}: \Omega(X) \longrightarrow \Omega(X)
$$

is continuous, and if $Y$ is another manifold (possibly with boundary) and if $\Psi$ : $X \rightarrow Y$ is a smooth map, then the pullback map

$$
\Psi^{*}: \Omega(Y) \longrightarrow \Omega(X)
$$

is continuous [17. 
For every continuous seminorm $\varepsilon$ on $\Omega(N)$ we get a seminorm $\varepsilon^{\mathbb{T}}$ on $\Omega_{\mathbb{T}}(N \times \mathbb{T})$ by setting

$$
\varepsilon^{\mathbb{T}}\left(\alpha+\vartheta_{\mathbb{T}} \wedge \beta\right):=\varepsilon(\alpha)+\varepsilon(\beta)
$$

In view of the formula $d_{\mathbb{T}}$, the space $\Omega_{\mathbb{T}}(N \times \mathbb{T})$ then becomes a locally convex unital DGA (by remark 3.1) in terms of the $\varepsilon^{\mathbb{T}}$ 's. As a consequence, we get the super complexes

$$
\begin{aligned}
& \mathscr{C}^{+}\left(\Omega_{\mathbb{T}}(N \times \mathbb{T})\right) \stackrel{b+B}{\longrightarrow} \mathscr{C}^{-}\left(\Omega_{\mathbb{T}}(N \times \mathbb{T})\right) \stackrel{b+B}{\longrightarrow} \mathscr{C}^{+}\left(\Omega_{\mathbb{T}}(N \times \mathbb{T})\right), \\
& \mathscr{N}^{+}\left(\Omega_{\mathbb{T}}(N \times \mathbb{T})\right) \stackrel{b+B}{\longrightarrow} \mathscr{N}^{-}\left(\Omega_{\mathbb{T}}(N \times \mathbb{T})\right) \stackrel{b+B}{\longrightarrow} \mathscr{N}^{+}\left(\Omega_{\mathbb{T}}(N \times \mathbb{T})\right), \\
& \mathscr{C}_{\epsilon}^{+}\left(\Omega_{\mathbb{T}}(N \times \mathbb{T})\right) \stackrel{b+B}{\longrightarrow} \mathscr{C}_{\epsilon}^{-}\left(\Omega_{\mathbb{T}}(N \times \mathbb{T})\right) \stackrel{b+B}{\longrightarrow} \mathscr{C}^{+}\left(\Omega_{\mathbb{T}}(N \times \mathbb{T})\right), \\
& \mathscr{N}_{\epsilon}^{+}\left(\Omega_{\mathbb{T}}(N \times \mathbb{T})\right) \stackrel{b+B}{\longrightarrow} \mathscr{N}_{\epsilon}^{-}\left(\Omega_{\mathbb{T}}(N \times \mathbb{T})\right) \stackrel{b+B}{\longrightarrow} \mathscr{N}_{\epsilon}^{+}\left(\Omega_{\mathbb{T}}(N \times \mathbb{T})\right) .
\end{aligned}
$$

\section{Equivariant Chen integrals}

Let us consider a compact manifold $N$ without boundary, and the space $L N$ of smooth loops $\gamma: \mathbb{T} \rightarrow N$, where in the sequel we read $\mathbb{T}$ as $\mathbb{T}=[0,1] / \sim$. This becomes an infinite dimensional Fréchet manifold which is locally modelled on the Fréchet space $L \mathbb{R}^{\operatorname{dim} N}$ of smooth loops $\mathbb{T} \rightarrow \mathbb{R}^{\operatorname{dim} N}$. Then $L N$ carries a natural smooth $\mathbb{T}$-action, given by rotating each loop, and the fixed point set of this action is precisely $N \subset L N$, embedded as constant loops. Given $\gamma \in L N$ the tangent space $T_{\gamma} L N$ is given by linear space of smooth vector fields on $N$ along $\gamma$, that is,

$$
T_{\gamma}(L N)=\left\{X \in C^{\infty}(\mathbb{T}, N): X(t) \in T_{\gamma(t)} N \text { for all } t \in \mathbb{T}\right\},
$$

and the generator of the $\mathbb{T}$-action on $L N$ is the vector field $\gamma \mapsto \dot{\gamma}$ on $L N$. Let $\iota$ denote the contraction with respect to the latter vector field. In the sequel, we understand

$$
\Omega(L N):=\bigoplus_{k=0}^{\infty} \Omega^{k}(L M) .
$$

For fixed $s \in \mathbb{T}$ one has the diffeomorphism

$$
\phi_{s}: L N \longrightarrow L N, \quad \gamma \longmapsto \gamma(s+\cdot)
$$

induced by the $\mathbb{T}$-action, and one gets an induced operator

$$
P: \Omega(L N) \longrightarrow \Omega(L N), \quad \text { defined on } \Omega^{k}(L N) \text { by } P \alpha:=\int_{0}^{1} \phi_{s}^{*} \iota \alpha d s .
$$

Then $P$ becomes a degree -1 derivation. In addition, there is the usual exterior derivative

$$
d: \Omega(L N) \longrightarrow \Omega(L N)
$$


a degree +1 derivation. Taking only odd/even degree forms, one gets the superstructure $\Omega=\Omega^{+}(L N) \oplus \Omega^{-}(L N)$, and we get the super complex

$$
\Omega^{+}(L N) \stackrel{d+P}{\longrightarrow} \Omega^{-}(L N) \stackrel{d+P}{\longrightarrow} \Omega^{+}(L N),
$$

called the equivariant de Rham complex of $L N$. This complex does not carry much information, as the differential forms of interest, like the Bismut-Chern character below, are actually elements of

$$
\prod_{k=0}^{\infty} \Omega^{k}(L N), \quad \text { rather than } \Omega(L N)=\bigoplus_{k=0}^{\infty} \Omega^{k}(L N) .
$$

Thus we are going to 'complete' $\Omega(L N)$ in some way. To this end, following Chen's approach [6] of constructing a smooth structure on $L N$ in terms of plots, we consider smooth maps $f: X \rightarrow L N$, where $X$ is a finite dimensional manifold (without boundary). Given a continuous seminorm $\varepsilon$ on $\Omega(X)$ we get an induced seminorm

$$
\varepsilon_{f}(\omega):=\varepsilon\left(f^{*} \omega\right) \quad \text { on } \Omega(L N) .
$$

The locally convex topology induced by the $\epsilon_{f}$ 's is Hausdorff and we define $\widehat{\Omega}(L N)$ to be the completion of $\Omega(L N)$ with respect to this locally convex topology. The maps $d, P$ and the grading operator become continuous maps $\Omega(L N) \rightarrow \Omega(L N)$ : indeed, the continuity of the grading map is trivial. The continuity of $d$ follows from

$$
\varepsilon_{f}(d \omega)=\varepsilon\left(d\left[f^{*} \omega\right]\right) \leq \varepsilon^{\prime}\left(f^{*} \omega\right)=\varepsilon_{f}^{\prime}(\omega)
$$

for some continuous seminorm $\varepsilon^{\prime}$ on $\Omega(X)$, where we have used the continuity of $d: \Omega(X) \rightarrow \Omega(X)$. Finally, the continuity of $P$ follows easily from the continuity of $\iota$, which in turn follows from writing

$$
\varepsilon_{f}(\iota \omega)=\varepsilon\left(f^{*}[\iota \omega]\right)=\varepsilon\left(r^{*} \iota_{\partial_{\amalg}} \hat{f}^{*} j^{*}[\omega]\right) \leq \varepsilon_{j \circ \hat{f}}^{\prime}(\omega)
$$

for some continuous seminorm $\varepsilon^{\prime}$ on $\Omega(X \times \mathbb{T})$, where

$$
r: X \longrightarrow X \times \mathbb{T}, \quad j: N \longrightarrow L N
$$

are the canonical embeddings, and

$$
\hat{f}: X \times \mathbb{T} \longrightarrow N
$$

the map induced by $f: X \rightarrow L N$, and where we have used Remark 3.1 (the continuity of $r^{*} \iota_{\partial_{\Psi}}$, which implies the existence of $\left.\varepsilon^{\prime}\right)$.

We end up with the super complex

$$
\widehat{\Omega}^{+}(L N) \stackrel{d+P}{\longrightarrow} \widehat{\Omega}^{-}(L N) \stackrel{d+P}{\longrightarrow} \widehat{\Omega}^{+}(L N),
$$

called the completed equivariant de Rham complex of $L N$. The corresponding homology groups are denoted by $\widehat{\mathrm{H}}_{\mathbb{T}}^{ \pm}(L N)$. 
Given $t \in \mathbb{T}$ and $\alpha \in \Omega^{k}(N)$ one denotes with $\alpha(t) \in \Omega^{k}(L N)$ the form obtained by pulling $\alpha$ back with respect to the evaluation map $\gamma \mapsto \gamma(t)$. With this notation at hand, one has the equivariant Chen integral map

$$
\rho: \mathscr{C}\left(\Omega_{\mathbb{T}}(N \times \mathbb{T})\right) \longrightarrow \Omega(L N)
$$

which is defined by

$$
\begin{aligned}
& \rho\left\langle\left(\alpha_{0}+\vartheta_{\mathbb{T}} \wedge \beta_{0}\right) \otimes \cdots \otimes\left(\alpha_{n}+\vartheta_{\mathbb{T}} \wedge \beta_{n}\right)\right\rangle \\
& :=\int_{0}^{1} d s \phi_{s}^{*} \int_{\Delta_{n}} \alpha_{0}(0) \wedge\left(\iota \alpha_{1}\left(t_{1}\right)-\beta_{1}\left(t_{1}\right)\right) \wedge \cdots \wedge\left(\iota \alpha_{n}\left(t_{n}\right)-\beta_{n}\left(t_{n}\right)\right) d t_{1} \cdots d t_{n},
\end{aligned}
$$

where

$$
\Delta_{n}=\left\{0 \leq t_{1} \leq \cdots \leq t_{n} \leq 1\right\} \subset \mathbb{R}^{n}
$$

denotes the standard $n$-simplex. We will also write

$$
\begin{aligned}
& \rho\left\langle\left(\alpha_{0}+\vartheta_{\mathbb{T}} \wedge \beta_{0}\right) \otimes \cdots \otimes\left(\alpha_{n}+\vartheta_{\mathbb{T}} \wedge \beta_{n}\right)\right\rangle \\
& =\int_{0}^{1} d s \phi_{s}^{*} \tilde{\rho}\left\langle\left(\alpha_{0}+\vartheta_{\mathbb{T}} \wedge \beta_{0}\right) \otimes \cdots \otimes\left(\alpha_{n}+\vartheta_{\mathbb{T}} \wedge \beta_{n}\right)\right\rangle .
\end{aligned}
$$

We collect the essential properties of $\rho$ in the following proposition:

Proposition 4.1. The map $\rho$ is a continuous morphism of super complexes

$$
\rho: \mathscr{C}\left(\Omega_{\mathbb{T}}(N \times \mathbb{T})\right) \longrightarrow \Omega(L N),
$$

which in turn descends to a continuous map of super complexes

$$
\rho: \mathscr{N}\left(\Omega_{\mathbb{T}}(N \times \mathbb{T})\right) \longrightarrow \Omega(L N) .
$$

In particular, by density, we obtain the continuous maps of super complexes

$$
\rho: \mathscr{C}_{\epsilon}\left(\Omega_{\mathbb{T}}(N \times \mathbb{T})\right) \longrightarrow \widehat{\Omega}(L N), \quad \rho: \mathscr{N}_{\epsilon}\left(\Omega_{\mathbb{T}}(N \times \mathbb{T})\right) \longrightarrow \widehat{\Omega}(L N) .
$$

Proof. i) The fact that (16) is a map of superspaces follows easily from observing that

$$
\begin{aligned}
\mathscr{C}^{+}\left(\Omega_{\mathbb{T}}(N \times \mathbb{T})\right) & =\bigoplus_{j=0}^{\infty} \mathscr{C}^{2 j}\left(\Omega_{\mathbb{T}}(N \times \mathbb{T})\right), \\
\mathscr{C}^{-}\left(\Omega_{\mathbb{T}}(N \times \mathbb{T})\right) & =\bigoplus_{j=0}^{\infty} \mathscr{C}^{2 j+1}\left(\Omega_{\mathbb{T}}(N \times \mathbb{T})\right),
\end{aligned}
$$

where

$$
\begin{aligned}
& \mathscr{C}^{k}\left(\Omega_{\mathbb{T}}(N \times \mathbb{T})\right) \\
& \left.=\bigoplus_{r=0}^{\infty} \bigoplus_{l_{0}+\cdots+l_{r}=k+r} \Omega_{\mathbb{T}}^{l_{0}}(N \times \mathbb{T})\right) \otimes \underline{\left.\Omega_{\mathbb{T}}^{l_{1}}(N \times \mathbb{T})\right)} \otimes \cdots \otimes \underline{\left.\Omega_{\mathbb{T}}^{l_{r}}(N \times \mathbb{T})\right)}
\end{aligned}
$$


and that $\rho$ maps $\mathscr{C}^{k}\left(\Omega_{\mathbb{T}}(N \times \mathbb{T})\right) \rightarrow \Omega^{k}(L N)$.

ii) Next we show that $\rho(b+B)=(d+P) \rho$. Setting $\omega_{j}=\alpha_{j}+\vartheta_{\mathbb{T}} \wedge \beta_{j}$, we first notice

$$
\begin{aligned}
\tilde{\rho} b\left\langle\omega_{0} \otimes \cdots \otimes \omega_{n}\right\rangle= & \tilde{\rho}\left\langle d_{\mathbb{T}} \omega_{0} \otimes \cdots \otimes \omega_{j-1} \otimes \omega_{j} \otimes \omega_{j+1} \otimes \cdots \otimes \omega_{n}\right\rangle \\
& -\tilde{\rho}\left\langle\sum_{j=1}^{n}(-1)^{r_{j-1}} \omega_{0} \otimes \cdots \otimes \omega_{j-1} \otimes d_{\mathbb{T}} \omega_{j} \otimes \omega_{j+1} \otimes \cdots \otimes \omega_{n}\right\rangle \\
& -\tilde{\rho}\left\langle\sum_{j=0}^{n-1}(-1)^{r_{j}} \omega_{0} \otimes \cdots \otimes \omega_{j-1} \otimes \omega_{j} \wedge \omega_{j+1} \otimes \omega_{j+2} \otimes \cdots \otimes \omega_{n}\right\rangle \\
& +(-1)^{\left(j_{n}-1\right) r_{n-1}} \tilde{\rho}\left\langle\omega_{n} \wedge \omega_{0} \otimes \omega_{1} \otimes \cdots \otimes \omega_{n-1}\right\rangle .
\end{aligned}
$$

The first two lines give

$$
\begin{aligned}
& \int_{\Delta_{n}}\left(d \alpha_{0}(0)+\beta_{0}(0)\right) \wedge\left(\iota \alpha_{1}\left(t_{1}\right)-\beta_{1}\left(t_{1}\right)\right) \wedge \cdots \wedge\left(\iota \alpha_{n}\left(t_{n}\right)-\beta_{n}\left(t_{n}\right)\right) d^{n} t \\
& -\sum_{j=1}^{n}(-1)^{r_{j-1}} \int_{\Delta_{n}} \alpha_{0}(0)\left(\iota \alpha_{1}\left(t_{1}\right)-\beta_{1}\left(t_{1}\right)\right) \wedge \cdots \wedge\left(\iota \alpha_{j-1}\left(t_{j-1}\right)-\beta_{j-1}\left(t_{j-1}\right)\right) \wedge \\
& \quad \wedge\left(\iota d \alpha_{j}\left(t_{j}\right)+\iota \beta_{j}\left(t_{j-1}\right)+d \beta_{j}\left(t_{j}\right)\right) \wedge\left(\iota \alpha_{j+1}\left(t_{j+1}\right)-\beta_{j+1}\left(t_{j+1}\right)\right) \wedge \cdots \wedge\left(\iota \alpha_{n}\left(t_{n}\right)-\beta_{n}\left(t_{n}\right)\right) d^{n} t,
\end{aligned}
$$

where $d^{n} t=d t_{1} \cdots d t_{n}$. Using that

$$
\Delta_{n}=\left\{\left(t_{1}, t_{2}, \ldots, t_{n}\right): 0 \leq t_{1} \leq \ldots \leq t_{j-1} \leq t_{j} \leq t_{j+1} \leq \ldots \leq t_{n}\right\},
$$

and that

$$
\iota d \alpha_{j}\left(t_{j}\right)=\frac{d}{d t_{j}} \alpha_{j}\left(t_{j}\right)-d \iota \alpha_{j}\left(t_{j}\right)
$$

it can be rewritten as

$$
\begin{aligned}
& \int_{\Delta_{n}}\left(d \alpha_{0}(0)+\beta_{0}(0)\right) \wedge\left(\iota \alpha_{1}\left(t_{1}\right)-\beta_{1}\left(t_{1}\right)\right) \wedge \cdots \wedge\left(\iota \alpha_{n}\left(t_{n}\right)-\beta_{n}\left(t_{n}\right)\right) d^{n} t \\
& +\sum_{j=1}^{n}(-1)^{r_{j-1}} \int_{\Delta_{n}} \alpha_{0}(0)\left(\iota \alpha_{1}\left(t_{1}\right)-\beta_{1}\left(t_{1}\right)\right) \wedge \cdots \wedge\left(\iota \alpha_{j-1}\left(t_{j-1}\right)-\beta_{j-1}\left(t_{j-1}\right)\right) \wedge \\
& \wedge d\left(\iota \alpha_{j}\left(t_{j}\right)-\beta_{j}\left(t_{j}\right)\right) \wedge\left(\iota \alpha_{j+1}\left(t_{j+1}\right)-\beta_{j+1}\left(t_{j+1}\right)\right) \wedge \cdots \wedge\left(\iota \alpha_{n}\left(t_{n}\right)-\beta_{n}\left(t_{n}\right)\right) d^{n} t \\
& -\sum_{j=1}^{n}(-1)^{r_{j-1}} \int_{\Delta_{n}} \alpha_{0}(0)\left(\iota \alpha_{1}\left(t_{1}\right)-\beta_{1}\left(t_{1}\right)\right) \wedge \cdots \wedge\left(\iota \alpha_{j-1}\left(t_{j-1}\right)-\beta_{j-1}\left(t_{j-1}\right)\right) \wedge \\
& \wedge \frac{d}{d t_{j}} \alpha_{j}\left(t_{j}\right) \wedge\left(\iota \alpha_{j+1}\left(t_{j+1}\right)-\beta_{j+1}\left(t_{j+1}\right)\right) \wedge \cdots \wedge\left(\iota \alpha_{n}\left(t_{n}\right)-\beta_{n}\left(t_{n}\right)\right) d^{n} t \\
& -\sum_{j=1}^{n}(-1)^{r_{j-1}} \int_{\Delta_{n}} \alpha_{0}(0)\left(\iota \alpha_{1}\left(t_{1}\right)-\beta_{1}\left(t_{1}\right)\right) \wedge \cdots \wedge\left(\iota \alpha_{j-1}\left(t_{j-1}\right)-\beta_{j-1}\left(t_{j-1}\right)\right) \wedge \\
& \quad \wedge \iota \beta_{j}\left(t_{j}\right) \wedge\left(\iota \alpha_{j+1}\left(t_{j+1}\right)-\beta_{j+1}\left(t_{j+1}\right)\right) \wedge \cdots \wedge\left(\iota \alpha_{n}\left(t_{n}\right)-\beta_{n}\left(t_{n}\right)\right) d^{n} t .
\end{aligned}
$$


The first two (three) lines give

$$
d \tilde{\rho}\left\langle\omega_{0} \otimes \cdots \otimes \omega_{n}\right\rangle+\int_{\Delta_{n}} \beta_{0}(0) \wedge\left(\iota \alpha_{1}\left(t_{1}\right)-\beta_{1}\left(t_{1}\right)\right) \wedge \cdots \wedge\left(\iota \alpha_{n}\left(t_{n}\right)-\beta_{n}\left(t_{n}\right)\right) d^{n} t, \quad \text { (19) }
$$

while the third (fourth and fifth) line can be integrated in $t_{j}$ from $t_{j-1}$ to $t_{j+1}$ thus getting

$$
\begin{aligned}
d \tilde{\rho}\left\langle\omega_{0} \otimes \cdots \otimes \omega_{n}\right\rangle+\int_{\Delta_{n}} \beta_{0}(0) \wedge\left(\iota \alpha_{1}\left(t_{1}\right)-\beta_{1}\left(t_{1}\right)\right) \wedge \cdots \wedge\left(\iota \alpha_{n}\left(t_{n}\right)-\beta_{n}\left(t_{n}\right)\right) d^{n} t \\
-\sum_{j=1}^{n-1}(-1)^{r_{j-1}} \int_{\Delta_{n-1}} \alpha_{0}(0) \wedge\left(\iota \alpha_{1}\left(t_{1}\right)-\beta_{1}\left(t_{1}\right)\right) \wedge \cdots \wedge\left(\iota \alpha_{j-1}\left(t_{j-1}\right)-\beta_{j-1}\left(t_{j-1}\right)\right) \wedge \\
\quad \wedge \alpha_{j}\left(t_{j+1}\right) \wedge\left(\iota \alpha_{j+1}\left(t_{j+1}\right)-\beta_{j+1}\left(t_{j+1}\right)\right) \wedge \cdots \wedge\left(\iota \alpha_{n}\left(t_{n}\right)-\beta_{n}\left(t_{n}\right)\right) d^{n} t_{j} \\
-(-1)^{r_{n-1}} \int_{\Delta_{n-1}} \alpha_{0}(0) \wedge\left(\iota \alpha_{1}\left(t_{1}\right)-\beta_{1}\left(t_{1}\right)\right) \wedge \cdots \wedge\left(\iota \alpha_{n-1}\left(t_{n-1}\right)-\beta_{n-1}\left(t_{n-1}\right)\right) \wedge \alpha_{n}(1) d^{n} t_{n} \\
+\sum_{j=2}^{n}(-1)^{r_{j-1}} \int_{\Delta_{n-1}} \alpha_{0}(0) \wedge\left(\iota \alpha_{1}\left(t_{1}\right)-\beta_{1}\left(t_{1}\right)\right) \wedge \cdots \wedge\left(\iota \alpha_{j-1}\left(t_{j-1}\right)-\beta_{j-1}\left(t_{j-1}\right)\right) \wedge \\
\quad \wedge \alpha_{j}\left(t_{j-1}\right) \wedge\left(\iota \alpha_{j+1}\left(t_{j+1}\right)-\beta_{j+1}\left(t_{j+1}\right)\right) \wedge \cdots \wedge\left(\iota \alpha_{n}\left(t_{n}\right)-\beta_{n}\left(t_{n}\right)\right) d^{n} t_{j} \\
+(-1)^{r_{0}} \int_{\Delta_{n-1}} \alpha_{0}(0) \wedge \alpha_{1}(0) \wedge\left(\iota \alpha_{2}\left(t_{2}\right)-\beta_{2}\left(t_{2}\right)\right) \wedge \cdots \wedge\left(\iota \alpha_{n}\left(t_{n}\right)-\beta_{n}\left(t_{n}\right)\right) d^{n} t_{1} \\
-\sum_{j=1}^{n}(-1)^{r_{j-1}} \int_{\Delta_{n}} \alpha_{0}(0) \wedge\left(\iota \alpha_{1}\left(t_{1}\right)-\beta_{1}\left(t_{1}\right)\right) \wedge \cdots \wedge\left(\iota \alpha_{j-1}\left(t_{j-1}\right)-\beta_{j-1}\left(t_{j-1}\right)\right) \wedge \\
\quad \wedge \iota \beta_{j}\left(t_{j}\right) \wedge\left(\iota \alpha_{j+1}\left(t_{j+1}\right)-\beta_{j+1}\left(t_{j+1}\right)\right) \wedge \cdots \wedge\left(\iota \alpha_{n}\left(t_{n}\right)-\beta_{n}\left(t_{n}\right)\right) d^{n} t
\end{aligned}
$$

where $d^{n} t_{j}=d t_{1} \cdots d t_{j-1} d t_{j+1} \cdots d t_{n}$. If in the fourth sum of integrals we change the summation variable from $j$ to $j+1$, then make the change of variable $t_{j} \rightarrow t_{j+1}$, and put it together with the second sum of integrals, after noting that $(-1)^{r_{j-1}}(-1)^{j_{j}}=-(-1)^{r_{j}}$, then summing the fourth and the second integrals we get

$$
\begin{aligned}
- & \sum_{j=1}^{n-1}(-1)^{r_{j-1}} \int_{\Delta_{n-1}} \alpha_{0}(0) \wedge\left(\iota \alpha_{1}\left(t_{1}\right)-\beta_{1}\left(t_{1}\right)\right) \wedge \cdots \wedge\left(\iota \alpha_{j-1}\left(t_{j-1}\right)-\beta_{j-1}\left(t_{j-1}\right)\right) \wedge \\
& \wedge\left[\alpha_{j}\left(t_{j+1}\right) \wedge\left(\iota \alpha_{j+1}\left(t_{j+1}\right)-\beta_{j+1}\left(t_{j+1}\right)\right)\right] \wedge \cdots \wedge\left(\iota \alpha_{n}\left(t_{n}\right)-\beta_{n}\left(t_{n}\right)\right) d^{n} t_{j} \\
+ & \sum_{j=1}^{n-1}(-1)^{r_{j}} \int_{\Delta_{n-1}} \alpha_{0}(0) \wedge\left(\iota \alpha_{1}\left(t_{1}\right)-\beta_{1}\left(t_{1}\right)\right) \wedge \cdots \wedge\left(\iota \alpha_{j-1}\left(t_{j-1}\right)-\beta_{j-1}\left(t_{j-1}\right)\right) \wedge \\
& \wedge\left[\left(\iota \alpha_{j}\left(t_{j+1}\right)-\beta_{j}\left(t_{j+1}\right)\right) \wedge \alpha_{j+1}\left(t_{j+1}\right)\right] \wedge \cdots \wedge\left(\iota \alpha_{n}\left(t_{n}\right)-\beta_{n}\left(t_{n}\right)\right) d^{n} t_{j} \\
= & \sum_{j=1}^{n-1}(-1)^{r_{j}} \int_{\Delta_{n-1}} \alpha_{0}(0) \wedge\left(\iota \alpha_{1}\left(t_{1}\right)-\beta_{1}\left(t_{1}\right)\right) \wedge \cdots \wedge\left(\iota \alpha_{j-1}\left(t_{j-1}\right)-\beta_{j-1}\left(t_{j-1}\right)\right) \wedge \\
& \wedge\left[\left(\iota \alpha_{j}\left(t_{j+1}\right)-\beta_{j}\left(t_{j+1}\right)\right) \wedge \alpha_{j+1}\left(t_{j+1}\right)+(-1)^{j_{j}-1} \alpha_{j}\left(t_{j+1}\right) \wedge\left(\iota \alpha_{j+1}\left(t_{j+1}\right)-\beta_{j+1}\left(t_{j+1}\right)\right)\right] \wedge \\
& \wedge \cdots \wedge\left(\iota \alpha_{n}\left(t_{n}\right)-\beta_{n}\left(t_{n}\right)\right) d^{n} t_{j} \\
= & \sum_{j=1}^{n-1}(-1)^{r_{j}} \tilde{\rho}\left\langle\omega_{0} \otimes \cdots \otimes \omega_{j-1} \otimes \omega_{j} \wedge \omega_{j+1} \otimes \omega_{j+2} \otimes \cdots \otimes \omega_{n}\right\rangle,
\end{aligned}
$$


which including the fifth integral in (4) becomes

$$
\tilde{\rho}\left\langle\sum_{j=0}^{n-1}(-1)^{r_{j}} \omega_{0} \otimes \cdots \otimes \omega_{j-1} \otimes \omega_{j} \wedge \omega_{j+1} \otimes \omega_{j+2} \otimes \cdots \otimes \omega_{n}\right\rangle .
$$

This cancels the second line of (18). After noting that $\alpha_{n}(1)=\alpha_{n}(0)$, we see that the third integral in (44) is just

$$
-(-1)^{\left(j_{n}-1\right) r_{n-1}} \tilde{\rho}\left\langle\omega_{n} \wedge \omega_{0} \otimes \omega_{1} \otimes \cdots \otimes \omega_{n-1}\right\rangle,
$$

which cancels the third line of (18). Thus, we get

$$
\begin{aligned}
\tilde{\rho} b & \left\langle\omega_{0} \otimes \cdots \otimes \omega_{n}\right\rangle=d \tilde{\rho}\left\langle\omega_{0} \otimes \cdots \otimes \omega_{n}\right\rangle \\
& +\int_{\Delta_{n}} \beta_{0}(0) \wedge\left(\iota \alpha_{1}\left(t_{1}\right)-\beta_{1}\left(t_{1}\right)\right) \wedge \cdots \wedge\left(\iota \alpha_{n}\left(t_{n}\right)-\beta_{n}\left(t_{n}\right)\right) d^{n} t \\
- & \sum_{j=1}^{n}(-1)^{r_{j-1}} \int_{\Delta_{n}} \alpha_{0}(0) \wedge\left(\iota \alpha_{1}\left(t_{1}\right)-\beta_{1}\left(t_{1}\right)\right) \wedge \cdots \wedge\left(\iota \alpha_{j-1}\left(t_{j-1}\right)-\beta_{j-1}\left(t_{j-1}\right)\right) \wedge \\
& \wedge \iota \beta_{j}\left(t_{j}\right) \wedge\left(\iota \alpha_{j+1}\left(t_{j+1}\right)-\beta_{j+1}\left(t_{j+1}\right)\right) \wedge \cdots \wedge\left(\iota \alpha_{n}\left(t_{n}\right)-\beta_{n}\left(t_{n}\right)\right) d^{n} t .
\end{aligned}
$$

Now, let us consider

$$
\begin{aligned}
& P \tilde{\rho}\left\langle\omega_{0} \otimes \cdots \otimes \omega_{n}\right\rangle= \int_{I} d s \phi_{s}^{*} \iota \int_{\Delta_{n}} \alpha_{0}(0) \wedge\left(\iota \alpha_{1}\left(t_{1}\right)-\beta_{1}\left(t_{1}\right)\right) \wedge \cdots \wedge\left(\iota \alpha_{n}\left(t_{n}\right)-\beta_{n}\left(t_{n}\right)\right) d^{n} t \\
&=\int_{I \times \Delta_{n}} \iota \alpha_{0}(s) \wedge\left(\iota \alpha_{1}\left(t_{1}+s\right)-\beta_{1}\left(t_{1}+s\right)\right) \wedge \cdots \wedge\left(\iota \alpha_{n}\left(t_{n}+s\right)-\beta_{n}\left(t_{n}+s\right)\right) d^{n} t d s \\
&-\sum_{j=1}^{n}(-1)^{r_{j-1}} \int_{I} d s \phi_{s}^{*} \int_{\Delta_{n}} \alpha_{0}(0) \wedge\left(\iota \alpha_{1}\left(t_{1}\right)-\beta_{1}\left(t_{1}\right)\right) \wedge \cdots \wedge\left(\iota \alpha_{j-1}\left(t_{j-1}\right)-\beta_{j-1}\left(t_{j-1}\right)\right) \wedge \\
& \wedge \iota \beta_{j}\left(t_{j}\right) \wedge\left(\iota \alpha_{j+1}\left(t_{j+1}\right)-\beta_{j+1}\left(t_{j+1}\right)\right) \wedge \cdots \wedge\left(\iota \alpha_{n}\left(t_{n}\right)-\beta_{n}\left(t_{n}\right)\right) d^{n} t,
\end{aligned}
$$

where now $I$ must be identified with the circle $\mathbb{T}$, and where we used that

$$
\iota\left(\iota \alpha_{k}\left(t_{k}\right)-\beta_{k}\left(t_{k}\right)\right)=-\iota \beta_{k}\left(t_{k}\right) .
$$

Now, for any given choice of $\bar{t}=\left(t_{1}, \ldots, t_{n}\right)$ such that $0 \leq t_{1} \leq \cdots \leq t_{n} \leq 1$, we can understand $\mathbb{T}$ as the union of almost everywhere $n+1$ disjoint intervals defined by

$$
I_{j}(\bar{t})=\left\{s \in \mathbb{T} \mid t_{j-1}+s \leq 1, t_{j}+s-1 \geq 0\right\}, \quad j=1, \ldots, n+1 .
$$

We see that

$$
D_{j}=\left\{I_{j}(\bar{t}) \times \bar{t} \mid \bar{t} \in \Delta_{n}\right\}
$$

is a $(n+1)$-simplex for any given $j$, and

$$
\bigcup_{j=1}^{n+1} D_{j}=I \times \Delta_{n}
$$


while $D_{j} \cap D_{k}$ has zero measure if $j \neq k$. Therefore,

$$
\begin{aligned}
& \int_{I \times \Delta_{n}} \iota \alpha_{0}(s) \wedge\left(\iota \alpha_{1}\left(t_{1}+s\right)-\beta_{1}\left(t_{1}+s\right)\right) \wedge \cdots \wedge\left(\iota \alpha_{n}\left(t_{n}+s\right)-\beta_{n}\left(t_{n}+s\right)\right) d^{n} t d s \\
& =\int_{I \times \Delta_{n}} \beta_{0}(s) \wedge\left(\iota \alpha_{1}\left(t_{1}+s\right)-\beta_{1}\left(t_{1}+s\right)\right) \wedge \cdots \wedge\left(\iota \alpha_{n}\left(t_{n}+s\right)-\beta_{n}\left(t_{n}+s\right)\right) d^{n} t d s \\
& +\int_{I \times \Delta_{n}}\left(\iota \alpha_{0}(s)-\beta_{0}\right) \wedge\left(\iota \alpha_{1}\left(t_{1}+s\right)-\beta_{1}\left(t_{1}+s\right)\right) \wedge \cdots \wedge\left(\iota \alpha_{n}\left(t_{n}+s\right)-\beta_{n}\left(t_{n}+s\right)\right) d^{n} t d s \\
& =\int_{I} d s \phi_{s}^{*} \int_{\Delta_{n}} \beta_{0}(0) \wedge\left(\iota \alpha_{1}\left(t_{1}\right)-\beta_{1}\left(t_{1}\right)\right) \wedge \cdots \wedge\left(\iota \alpha_{n}\left(t_{n}\right)-\beta_{n}\left(t_{n}\right)\right) d^{n} t d s \\
& +\sum_{j=1}^{n+1} \int_{D_{j}}\left(\iota \alpha_{0}(s)-\beta_{0}(s)\right) \wedge\left(\iota \alpha_{1}\left(t_{1}+s\right)-\beta_{1}\left(t_{1}+s\right)\right) \wedge \cdots \wedge\left(\iota \alpha_{n}\left(t_{n}+s\right)-\beta_{n}\left(t_{n}+s\right)\right) d^{n} t d s .
\end{aligned}
$$

Now, for any given $j$ we introduce the variables

$$
\begin{aligned}
\tau_{k} & =t_{j+k-1}+s-1, \quad k=1, \ldots, n+1-j, \\
\tau_{n+2-j} & =s \\
\tau_{k} & =t_{k+j-n-2}+s, \quad k=n+3-j, \ldots, n+1 \quad(\text { if } j \geq 2) .
\end{aligned}
$$

In this coordinates we have

$$
D_{j}=\left\{\left(\tau_{1}, \ldots, \tau_{n+1}\right) \mid 0 \leq \tau_{1} \leq \cdots \leq \tau_{n+1} \leq 1\right\} \equiv \Delta_{n+1}, \quad d^{n} t d s=d^{n+1} \tau,
$$

and

$$
\begin{aligned}
& \left(\iota \alpha_{0}(s)-\beta_{0}(s)\right) \wedge\left(\iota \alpha_{1}\left(t_{1}+s\right)-\beta_{1}\left(t_{1}+s\right)\right) \wedge \cdots \wedge\left(\iota \alpha_{n}\left(t_{n}+s\right)-\beta_{n}\left(t_{n}+s\right)\right) \\
& =(-1)^{r_{j-1}\left(r_{n}-r_{j}\right)} 1 \wedge\left(\iota \alpha_{j}\left(\tau_{1}\right)-\beta_{j}\left(\tau_{1}\right)\right) \wedge \cdots \wedge\left(\iota \alpha_{n}\left(\tau_{n-j+1}\right)-\beta_{n}\left(\tau_{n-j+1}\right)\right) \wedge \\
& \quad \wedge\left(\iota \alpha_{0}\left(\tau_{n-j+2}\right)-\beta_{0}\left(\tau_{n-j+2}\right)\right) \wedge \cdots \wedge\left(\iota \alpha_{j-1}\left(\tau_{n+1}\right)-\beta_{j-1}\left(\tau_{n+1}\right)\right) .
\end{aligned}
$$

Integrating over $D_{j}=\Delta_{n+1}$ it becomes

$$
\begin{aligned}
& \int_{D_{j}}\left(\iota \alpha_{0}(s)-\beta_{0}(s)\right) \wedge\left(\iota \alpha_{1}\left(t_{1}+s\right)-\beta_{1}\left(t_{1}+s\right)\right) \wedge \cdots \wedge\left(\iota \alpha_{n}\left(t_{n}+s\right)-\beta_{n}\left(t_{n}+s\right)\right) \\
& =\rho\left\langle(-1)^{r_{j-1}\left(r_{n}-r_{j}\right)} 1 \otimes \omega_{j} \otimes \cdots \otimes \omega_{n} \otimes \omega_{0} \otimes \cdots \otimes \omega_{j-1}\right\rangle,
\end{aligned}
$$

and after summation over $j$ we finally get

$P \tilde{\rho}\left\langle\omega_{0} \otimes \cdots \otimes \omega_{n}\right\rangle=\tilde{\rho} B\left\langle\omega_{0} \otimes \cdots \otimes \omega_{n}\right\rangle$

$+\int_{I} d s \phi_{s}^{*} \int_{\Delta_{n}} \beta_{0}(0) \wedge\left(\iota \alpha_{1}\left(t_{1}\right)-\beta_{1}\left(t_{1}\right)\right) \wedge \cdots \wedge\left(\iota \alpha_{n}\left(t_{n}\right)-\beta_{n}\left(t_{n}\right)\right) d^{n} t d s$

$-\sum_{j=1}^{n}(-1)^{r_{j-1}} \int_{I} d s \phi_{s}^{*} \int_{\Delta_{n}} \alpha_{0}(0) \wedge\left(\iota \alpha_{1}\left(t_{1}\right)-\beta_{1}\left(t_{1}\right)\right) \wedge \cdots \wedge\left(\iota \alpha_{j-1}\left(t_{j-1}\right)-\beta_{j-1}\left(t_{j-1}\right)\right) \wedge$

$\wedge \iota \beta_{j}\left(t_{j}\right) \wedge\left(\iota \alpha_{j+1}\left(t_{j+1}\right)-\beta_{j+1}\left(t_{j+1}\right)\right) \wedge \cdots \wedge\left(\iota \alpha_{n}\left(t_{n}\right)-\beta_{n}\left(t_{n}\right)\right) d^{n} t$.

Notice that the second and third lines here are the means over $\mathbb{T}$ of the corresponding terms in (21). After taking the mean of both expressions and subtracting each other, we finally get $\rho(b+B)=(d+P) \rho$ as desired. 
iii) We now prove that $\tilde{\rho}$ vanishes on $\mathscr{D}\left(\Omega_{\mathbb{T}}(N \times \mathbb{T})\right)$. This implies that $\rho$ vanishes on $\mathscr{D}\left(\Omega_{\mathbb{T}}(N \times \mathbb{T})\right)$, too. For elements of the form (4) the assertion immediately follows from the fact that $\iota f(t)=0$, as $f(t)$ is a zero form. So, let us consider an element of the form (5). Since (recall that $f$ is constant over $\mathbb{T}$ )

$$
\iota d f(t)=\frac{d}{d t} f(t),
$$

and $d f=d_{\mathbb{T}} f$, we can write

$$
\begin{aligned}
& \tilde{\rho}\left(\left\langle\omega_{0} \otimes \cdots \otimes \omega_{r-1} f \otimes \omega_{r+1} \otimes \cdots \otimes \omega_{n}\right\rangle+\left\langle\omega_{0} \otimes \cdots \otimes \omega_{r-1} \otimes d f \otimes \omega_{r+1} \otimes \cdots \otimes \omega_{n}\right\rangle\right. \\
& \left.\quad-\left\langle\omega_{0} \otimes \cdots \otimes \omega_{r-1} \otimes f \omega_{r+1} \otimes \cdots \otimes \omega_{n}\right\rangle\right) \\
& =\int_{\Delta_{n-1}} \alpha_{0}(0) \wedge \cdots \wedge\left(\iota \alpha_{r-1}\left(t_{r-1}\right) f\left(t_{r-1}\right)-\beta_{r-1}\left(t_{r-1}\right) f\left(t_{r-1}\right)\right) \wedge\left(\iota \alpha_{r+1}\left(t_{r+1}\right)-\beta_{r+1}\left(t_{r+1}\right)\right) \wedge \\
& \quad \wedge \cdots \wedge\left(\iota \alpha_{n}\left(t_{n}\right)-\beta_{n}\left(t_{n}\right)\right) d^{n} t_{r} \\
& -\int_{\Delta_{n-1}} \alpha_{0}(0) \wedge \cdots \wedge\left(\iota \alpha_{r-1}\left(t_{r-1}\right)-\beta_{r-1}\left(t_{r-1}\right)\right) \wedge\left(f\left(t_{r+1}\right) \iota \alpha_{r+1}\left(t_{r+1}\right)-f\left(t_{r+1}\right) \beta_{r+1}\left(t_{r+1}\right)\right) \wedge \\
& \quad \wedge \cdots \wedge\left(\iota \alpha_{n}\left(t_{n}\right)-\beta_{n}\left(t_{n}\right)\right) d^{n} t_{r} \\
& +\int_{\Delta_{n}} \alpha_{0}(0) \wedge \cdots \wedge\left(\iota \alpha_{r-1}\left(t_{r-1}\right)-\beta_{r-1}\left(t_{r-1}\right)\right) \wedge \frac{d}{d t_{r}} f\left(t_{r}\right) \wedge\left(\iota \alpha_{r}\left(t_{r}\right)-\beta_{r}\left(t_{r}\right)\right) \wedge \\
& \quad \wedge \cdots \wedge\left(\iota \alpha_{n}\left(t_{n}\right)-\beta_{n}\left(t_{n}\right)\right) d^{n} t .
\end{aligned}
$$

After integrating $t_{r}$ from $t_{r-1}$ to $t_{r+1}$ in the last term, we get exactly zero.

v) It remains to check the continuity of (16), which easily follow from the continuity of $\tilde{\rho}$. To see the latter, let $X$ be a smooth manifold (without boundary), let $\varepsilon$ be a continuous seminorm on $\Omega(X)$, and let $f: X \rightarrow L N$ be smooth. For $s \in \mathbb{T}$ let $r_{s}$ denote the embedding

$$
X \longrightarrow X \times \mathbb{T}, x \longmapsto(x, s) .
$$

Then we have

$$
\begin{aligned}
& \varepsilon_{f}\left(\tilde{\rho}\left\langle\left(\alpha_{0}+\vartheta_{\mathbb{T}} \wedge \beta_{0}\right) \otimes \cdots \otimes\left(\alpha_{n}+\vartheta_{\mathbb{T}} \wedge \beta_{n}\right)\right\rangle\right) \\
& \leq \int_{\Delta_{n}} \varepsilon\left(f^{*}\left[\alpha_{0}(0)\right]\right) \prod_{i=1}^{n} \varepsilon\left(f^{*}\left[\iota \alpha_{i}\left(t_{i}\right)-\beta_{i}\left(t_{i}\right)\right]\right) d t_{1} \cdots d t_{n} \\
& =\int_{\Delta_{n}} \varepsilon\left(r_{0}^{*} \hat{f}^{*} \alpha_{0}\right) \prod_{i=1}^{n} \varepsilon\left(r_{t_{i}}^{*} \iota \partial_{\mathbb{T}} \hat{f}^{*} \alpha_{i}-r_{t_{i}}^{*} \hat{f}^{*} \beta_{i}\right) d t_{1} \cdots d t_{n} \\
& \leq \int_{\Delta_{n}} \varepsilon\left(r_{0}^{*} \hat{f}^{*} \alpha_{0}\right) \prod_{i=1}^{n}\left(\varepsilon\left(r_{t_{i}}^{*} \iota_{\partial_{\mathbb{T}}} \hat{f}^{*} \alpha_{i}\right)+\varepsilon\left(r_{t_{i}}^{*} \hat{f}^{*} \beta_{i}\right)\right) d t_{1} \cdots d t_{n} \\
& \leq \int_{\Delta_{n}} \tilde{\varepsilon}\left(\alpha_{0}\right) \prod_{i=1}^{n}\left(\tilde{\varepsilon}\left(\alpha_{i}\right)+\tilde{\varepsilon}\left(\beta_{i}\right)\right) d t_{1} \cdots d t_{n} \\
& \leq \frac{1}{n !} \prod_{i=0}^{n}\left(\tilde{\varepsilon}\left(\alpha_{i}\right)+\tilde{\varepsilon}\left(\beta_{i}\right)\right)=\frac{1}{n !} \tilde{\varepsilon}_{n}^{\mathbb{T}}\left(\left(\alpha_{0}+\vartheta_{\mathbb{T}} \wedge \beta_{0}\right) \otimes \cdots \otimes\left(\alpha_{n}+\vartheta_{\mathbb{T}} \wedge \beta_{n}\right)\right),
\end{aligned}
$$


for some continuous seminorm $\tilde{\varepsilon}$ on $\Omega(N)$. This estimate shows the continuity of $\tilde{\rho}$ and completes the proof.

\section{Construction of cycles in $\mathscr{N}_{\epsilon}^{-}\left(\Omega_{\mathbb{T}}(M \times \mathbb{T})\right)$ and the induced cycles in $\widehat{\Omega}^{-}(L M)$}

Let now $M$ be a compact manifold (possibly with boundary). Given $g \in C^{\infty}(M, U(l \times$ $l ; \mathbb{C})$ ) our aim is to construct a canonically given element

$$
\mathrm{Ch}^{-}(g) \in \mathscr{C}_{\epsilon}^{-}\left(\Omega_{\mathbb{T}}(M \times \mathbb{T})\right)
$$

with $(b+B) \mathrm{Ch}^{-}(g)=0$ in the Chen normalized complex. To this end, let $I:=[0,1]$ and denote the canonical vector field on $I$ with $\partial_{I}$. We denote the canonical Maurer-Cartan form on $U(l \times l ; \mathbb{C})$ by

$$
\omega \in \Omega^{1}(U(l \times l ; \mathbb{C}), \operatorname{Mat}(l \times l ; \mathbb{C})) .
$$

Then for all $s \in I$ we can form the covariant derivative $d+s \omega$ on the trivial vector bundle $U(l \times l ; \mathbb{C}) \times \mathbb{C}^{l} \rightarrow U(l \times l ; \mathbb{C})$. Let

$$
A^{s} \in \Omega^{1}(U(l \times l ; \mathbb{C}), \operatorname{Mat}(l \times l ; \mathbb{C})), \quad R^{s} \in \Omega^{2}(U(l \times l ; \mathbb{C}), \operatorname{Mat}(l \times l ; \mathbb{C}))
$$

denote the connection 1-form of $d+s \omega$ and the curvature of $d+s \omega$, respectively, and

$$
\mathcal{A}^{s}:=A^{s}-\vartheta_{\mathbb{T}} \wedge R^{s} \in \Omega_{\mathbb{T}}(U(l \times l ; \mathbb{C}) \times \mathbb{T}, \operatorname{Mat}(l \times l ; \mathbb{C})) .
$$

We set

$$
A^{s}(g):=g^{*} A^{s}, \quad R_{g}^{s}:=g^{*} R^{s}, \quad \omega_{g}:=g^{*} \omega,
$$

so that $A^{s}(g)=s \omega_{g}$ and by the Maurer-Cartan equation $R_{g}^{s}=(s / 2) \omega_{g}^{2}$. Then we can define

$$
\mathcal{A}^{s}(g):=A_{g}^{s}-\vartheta_{\mathbb{T}} \wedge R_{g}^{s} \in \Omega_{\mathbb{T}}(M \times \mathbb{T}, \operatorname{Mat}(l \times l ; \mathbb{C})) .
$$

By varying $s$, the forms $\mathcal{A}^{s}(g)$ induce a form

$$
\mathcal{A}(g) \in \Omega_{\mathbb{T}}(M \times I \times \mathbb{T}, \operatorname{Mat}(l \times l ; \mathbb{C}))
$$

and we set

$$
\mathcal{B}(g):=\iota_{\partial_{I}} \mathcal{A}(g) \in \Omega_{\mathbb{T}}(M \times I \times \mathbb{T}, \operatorname{Mat}(l \times l ; \mathbb{C})) .
$$

Then we can define

$$
\mathcal{B}^{s}(g) \in \Omega_{\mathbb{T}}(M \times \mathbb{T}, \operatorname{Mat}(l \times l ; \mathbb{C})),
$$

to be the pullback of $\mathcal{B}(g)$ with respect to the embedding

$$
M \times \mathbb{T} \longrightarrow M \times I \times \mathbb{T}, \quad(x, t) \longmapsto(x, s, t) .
$$


In fact, by a simple calculation one finds

$$
\mathcal{A}^{s}(g)=s \omega_{g}+s(1-s) \vartheta_{\mathbb{T}} \wedge \omega_{g}^{2}, \quad \mathcal{B}^{s}(g)=-\vartheta_{\mathbb{T}} \wedge \omega_{g},
$$

so that $\mathcal{B}^{s}(g)$ actually does not depend on $s$. With these preparations, we can define an element

$$
\mathrm{Ch}^{-}(g)=\left(\mathrm{Ch}_{0}^{-}(g), \mathrm{Ch}_{1}^{-}(g), \ldots\right) \in \mathscr{C}\left(\Omega_{\mathbb{T}}(M \times \mathbb{T})\right)
$$

by setting

$$
\mathrm{Ch}_{n}^{-}(g):=\operatorname{Tr}_{n}\left[\int_{0}^{1} 1 \otimes \sum_{k=1}^{n} \mathcal{A}^{s}(g)^{\otimes(k-1)} \otimes \mathcal{B}^{s}(g) \otimes \mathcal{A}^{s}(g)^{\otimes(n-k)} d s\right],
$$

where given linear spaces $V_{0}, \ldots, V_{n}$, and $v^{(j)} \in \operatorname{Mat}\left(l \times l ; V_{i}\right), j=0, \ldots, n$, the generalized trace is defined by

$$
\operatorname{Tr}_{n}\left[v^{(0)} \otimes \cdots \otimes v^{(n)}\right]:=\sum_{i_{0}, \ldots, i_{n}=1, \ldots l} v_{i_{0}, i_{1}}^{(0)} \otimes v_{i_{1}, i_{2}}^{(1)} \otimes \cdots \otimes v_{i_{n}, i_{0}}^{(n)} .
$$

We refer the reader to the paper 15] by Simons and Sullivan, where a construction of the usual odd Chern character $\mathrm{ch}^{-}(g) \in \Omega^{-}(M)$ (cf. formula (24) below) has been given that influenced our definition of $\mathrm{Ch}^{-}(g)$.

Theorem 5.1. Let $M$ be a compact manifold, possibly with boundary.

a) One has

$$
\mathrm{Ch}^{-}(g) \in \mathscr{C}_{\epsilon}^{-}\left(\Omega_{\mathbb{T}}(M \times \mathbb{T})\right), \quad \text { and }(b+B) \mathrm{Ch}^{-}(g)=0 \text { in } \mathscr{N}_{\epsilon}\left(\Omega_{\mathbb{T}}(M \times \mathbb{T})\right),
$$

in particular, $\mathrm{Ch}^{-}(g)$ induces a homology class

$$
\left[\mathrm{Ch}^{-}(g)\right] \in \mathrm{HN}_{\epsilon}^{-}\left(\Omega_{\mathbb{T}}(M \times \mathbb{T})\right) .
$$

b) The map

$$
\mathrm{K}^{-1}(M) \longrightarrow \mathrm{HN}_{\epsilon}^{-}\left(\Omega_{\mathbb{T}}(M \times \mathbb{T})\right), \quad[g] \longmapsto\left[\mathrm{Ch}^{-}(g)\right]
$$

is a well-defined group homomorphism.

Proof. a) It is easily seen that $\Gamma \mathrm{Ch}^{-}(g)=-\mathrm{Ch}^{-}(g)$. To show that

$$
\mathrm{Ch}^{-}(g) \in \mathscr{C}_{\epsilon}^{-}\left(\Omega_{\mathbb{T}}(M \times \mathbb{T})\right),
$$

given a continuous seminorm $\varepsilon$ on $\Omega_{\mathbb{T}}(M \times \mathbb{T})$ set

$$
C_{\varepsilon}:=\sup _{s \in[0,1]} \max \left(\varepsilon(1), \max _{i, j=1, \ldots, l} \varepsilon\left(\mathcal{A}^{s}(g)_{i j}\right), \max _{i, j=1, \ldots, l} \varepsilon\left(\mathcal{B}^{s}(g)_{i j}\right)\right) .
$$

It is then easily checked that

$$
\kappa_{\varepsilon}\left(\mathrm{Ch}^{-}(g)\right) \leq \sum_{n=0}^{\infty} n \frac{\left(l^{2} C_{\varepsilon}\right)^{n}}{\sqrt{n !}}<\infty .
$$


It remains to prove

$$
(b+B) \mathrm{Ch}^{-}(g) \in \mathscr{D}_{\epsilon}\left(\Omega_{\mathbb{T}}(M \times \mathbb{T})\right) .
$$

In fact,

$$
B \mathrm{Ch}^{-}(g) \in \mathscr{D}_{\epsilon}\left(\Omega_{\mathbb{T}}(M \times \mathbb{T})\right),
$$

as every $\left\langle\mathrm{Ch}_{n}^{-}(g)\right\rangle$ contains the 0 -form 1 and so is of the form (4) with $f=1$. It remains to show that

$$
b \mathrm{Ch}^{-}(g) \in \mathscr{D}_{\epsilon}\left(\Omega_{\mathbb{T}}(M \times \mathbb{T})\right) .
$$

In order to see the latter, let us first notice that

$$
\left(b \mathrm{Ch}^{-}(g)\right)_{n}=\left(b\left\langle\mathrm{Ch}_{n}^{-}(g)\right\rangle\right)_{n}+\left(b\left\langle\mathrm{Ch}_{n+1}^{-}(g)\right\rangle\right)_{n} .
$$

Using (23) and the explicit definition of $b$, we get

$$
\begin{aligned}
& \left(b\left\langle\mathrm{Ch}_{n}^{-}(g)\right\rangle\right)_{n} \\
& =-\operatorname{Tr}_{n}\left[\int_{0}^{1} 1 \otimes \sum_{k=1}^{n} \sum_{l=0}^{k-2} \mathcal{A}^{s}(g)^{\otimes l} \otimes\left(-s^{2} \omega_{g}^{2}\right) \otimes \mathcal{A}^{s}(g)^{\otimes(k-l-2)}\right. \\
& \quad+\operatorname{Tr}_{n}\left[\int_{0}^{1} 1 \otimes \sum_{k=1}^{n} \sum_{l=0}^{n-k-1} \mathcal{A}_{\mathbb{T}}^{s}(g)^{\otimes(k-1)} \otimes\left(-\vartheta_{\mathbb{T}} \wedge \omega_{g}\right)\right. \\
& -\operatorname{Tr}_{n}\left[\int_{0}^{1} 1 \otimes \sum_{k=1}^{n} \mathcal{A}^{s}(g)^{\otimes(n-k)} d s\right]
\end{aligned}
$$

and

$$
\begin{aligned}
& \left(b\left\langle\mathrm{Ch}_{n+1}^{-}(g)\right\rangle\right)_{n} \\
& =-\operatorname{Tr}_{n}\left[\int_{0}^{1} 1 \otimes \sum_{k=1}^{n} \sum_{l=0}^{k-2} \mathcal{A}^{s}(g)^{\otimes l} \otimes\left(+s^{2} \omega_{g}^{2}\right) \otimes \mathcal{A}^{s}(g)^{\otimes(k-l-2)}\right. \\
& \quad+\operatorname{Tr}_{n}\left[\int_{0}^{1} 1 \otimes \sum_{k=1}^{n} \sum_{l=0}^{n-k-1} \mathcal{A}^{s}(g)^{\otimes(k-1)} \otimes\left(-\omega_{\mathrm{T}} \wedge \mathcal{A}^{s}(g)^{\otimes(n-k)} d s\right] \otimes \mathcal{A}^{s}(g)^{\otimes l}\right. \\
& \quad-\operatorname{Tr}_{n}\left[\int_{0}^{1} 1 \otimes \sum_{k=1}^{n} \mathcal{A}^{s}(g)^{\otimes(k-1)} \otimes\left(-2 s \vartheta_{\mathbb{T}} \wedge \omega_{g}^{2}\right) \otimes \mathcal{A}^{s}(g)^{\otimes(n-k)} d s\right],
\end{aligned}
$$


whose sum is

$$
\begin{aligned}
& \operatorname{Tr}_{n}\left[\int_{0}^{1} 1 \otimes \sum_{k=1}^{n} \mathcal{A}^{s}(g)^{\otimes(k-1)} \otimes\left(\frac{d}{d s} \mathcal{A}^{s}(g)\right) \otimes \mathcal{A}^{s}(g)^{\otimes(n-k)} d s\right] \\
& =\operatorname{Tr}_{n}\left[\int_{0}^{1} \frac{d}{d s}\left(1 \otimes \mathcal{A}^{s}(g)^{\otimes n}\right) d s\right]=\operatorname{Tr}_{n}\left[1 \otimes \mathcal{A}^{1}(g)^{\otimes n}\right]-\operatorname{Tr}_{n}\left[1 \otimes \mathcal{A}^{0}(g)^{\otimes n}\right] .
\end{aligned}
$$

Thus, we finally have

$$
\left(b \mathrm{Ch}^{-}(g)\right)_{n}=\operatorname{Tr}_{n}\left[1 \otimes \omega_{g}^{\otimes n}\right], \quad n=1,2, \ldots .
$$

We now prove that

$$
\left(\ldots, \operatorname{Tr}_{n}\left[1 \otimes \omega_{g}^{\otimes n}\right], \ldots\right) \in \mathscr{D}_{\epsilon}\left(\Omega_{\mathbb{T}}(M \times \mathbb{T})\right) .
$$

To this end we have simply to employ the properties of the generalized trace. Indeed, for $n \geq 2$ we can write

$$
\begin{aligned}
\left\langle\operatorname{Tr}_{n}\left[1 \otimes \omega_{g}^{\otimes n}\right]\right\rangle= & \left\langle\operatorname{Tr}_{n}\left[1 \otimes \omega_{g} \otimes \omega_{g} \otimes \omega_{g}^{\otimes(n-2)}\right]\right\rangle \\
= & -\left\langle\operatorname{Tr}_{n}\left[1 \otimes d g^{-1} \otimes d g \otimes \omega_{g}^{\otimes(n-2)}\right]\right\rangle \\
= & -\left\langle\operatorname{Tr}_{n}\left[1 \otimes d g^{-1} \otimes d g \otimes \omega_{g}^{\otimes(n-2)}\right]\right\rangle \\
& -\left\langle\operatorname{Tr}_{n-1}\left[g^{-1} \otimes d g \otimes \omega_{g}^{\otimes(n-2)}\right]\right\rangle \\
& +\left\langle\operatorname{Tr}_{n-1}\left[1 \otimes g^{-1} d g \otimes \omega_{g}^{\otimes(n-2)}\right]\right\rangle
\end{aligned}
$$

where the last two terms cancel each other because of the trace property, which is precisely of the form (5) for $f=g^{-1}$. Similarly, for $n=1$ it is sufficient to notice that

$$
\left\langle\operatorname{Tr}_{1}\left[1 \otimes \omega_{g}\right]\right\rangle=\left\langle\operatorname{Tr}_{1}\left[g^{-1} \otimes d g\right]\right\rangle,
$$

which is of the form (4) with $f=g^{-1}$, completing the proof of $b \mathrm{Ch}^{-}(g) \in$ $\mathscr{D}_{\epsilon}\left(\Omega_{\mathbb{T}}(M \times \mathbb{T})\right)$.

b) It suffices to prove the following two facts:

i) If $g, h \in C^{\infty}(M, U(l \times l ; \mathbb{C}))$, then one has $\mathrm{Ch}^{-}(g \oplus h)=\mathrm{Ch}^{-}(g)+\mathrm{Ch}^{-}(h)$.

ii) If $g_{0}, g_{1} \in C^{\infty}(M, U(l \times l ; \mathbb{C}))$ are connected by a smooth homotopy

$$
\text { g. } \in C^{\infty}(M \times I, U(l \times l ; \mathbb{C})),
$$

then one has

$$
\mathrm{Ch}^{-}\left(g_{1}\right)-\mathrm{Ch}^{-}\left(g_{0}\right)=(b+B) w \quad \text { in } \mathscr{N}_{\epsilon}\left(\Omega_{\mathbb{T}}(M \times \mathbb{T})\right)
$$

for some $w \in \mathscr{C}_{\epsilon}\left(\Omega_{\mathbb{T}}(M \times \mathbb{T})\right)$.

Here, property i) is an immediate consequence of the properties of the generalized 
trace $\mathrm{T}_{n}$ using the block diagonal form of $g \oplus h$.

To see ii), for any $t \in I$, we define the embedding

$$
j_{t}: M \hookrightarrow M \times I, \quad x \longmapsto(x, t),
$$

and $w=\left(w_{0}, w_{1}, \ldots\right) \in \mathscr{C}_{\epsilon}\left(\Omega_{\mathbb{T}}(M \times \mathbb{T})\right)$ by setting

$$
\begin{aligned}
w_{n}:= & -\operatorname{Tr}_{n}\left[\int _ { 0 } ^ { 1 } \int _ { 0 } ^ { 1 } 1 \otimes \sum _ { k = 1 } ^ { n } \sum _ { l = 0 } ^ { k - 2 } j _ { t } ^ { * } \left(\mathcal{A}^{s}(g .)^{\otimes l} \otimes \iota_{\partial_{I}} \mathcal{A}^{s}(g .) \otimes \mathcal{A}^{s}(g .)^{\otimes(k-l-2)}\right.\right. \\
& \left.\left.\otimes \mathcal{B}^{s}(g .) \otimes \mathcal{A}^{s}(g .)^{\otimes(n-k)}\right) d s d t\right] \\
& +\operatorname{Tr}_{n}\left[\int _ { 0 } ^ { 1 } \int _ { 0 } ^ { 1 } 1 \otimes \sum _ { k = 1 } ^ { n } \sum _ { l = 0 } ^ { n - k - 1 } j _ { t } ^ { * } \left(\mathcal{A}^{s}(g .)^{\otimes(k-1)} \otimes \mathcal{B}^{s}(g .) \otimes \mathcal{A}^{s}(g .)^{\otimes l} \otimes \iota_{\partial_{I}} \mathcal{A}^{s}(g .)\right.\right. \\
& \left.\left.\otimes \mathcal{A}^{s}(g .)^{\otimes(n-k-l-1)}\right) d s d t\right] \\
& -\operatorname{Tr}_{n}\left[\int_{0}^{1} \int_{0}^{1} 1 \otimes \sum_{k=1}^{n} j_{t}^{*}\left(\mathcal{A}^{s}(g .)^{\otimes(k-1)} \otimes \iota_{\partial_{I}} \mathcal{B}^{s}(g .) \otimes \mathcal{A}^{s}(g .)^{\otimes(n-k)}\right) d s d t\right] .
\end{aligned}
$$

The $\mathscr{C}_{\epsilon}$ growth conditions are easily checked for $w$. Then again it is clear that $B w \in \mathscr{D}_{\epsilon}\left(\Omega_{\mathbb{T}}(M \times \mathbb{T})\right)$. On the other hand, by using the identity

$$
d j_{t}^{*} \iota_{\partial_{I}} \mathcal{A}^{s}(g .)=-j_{t}^{*} \iota_{\partial_{I}} d \mathcal{A}^{s}(g .)+\frac{\partial}{\partial t} j_{t}^{*} \mathcal{A}^{s}(g .),
$$

and similarly for $\mathcal{B}^{s}$, and the same computations as in part a) we get, as elements in the Chen normalized complex,

$$
\begin{aligned}
(b w+B w)_{n} & =(b w)_{n}=\left(b\left\langle w_{n}\right\rangle\right)_{n}+\left(b\left\langle w_{n+1}\right\rangle\right)_{n}=\left(\left\langle\int_{0}^{1} \frac{d}{d t} j_{t}^{*} \mathrm{Ch}^{-}(g .)\right)_{n}\right. \\
& =\mathrm{Ch}_{n}^{-}\left(g_{1}\right)-\mathrm{Ch}_{n}^{-}\left(g_{0}\right) .
\end{aligned}
$$

This completes the proof.

If $M$ has no boundary (so that $L M$ is a well-defined Fréchet manifold), in view of $(d+P) \rho=\rho(b+B)$, we immediately get:

Corollary 5.2. Assume $M$ is a compact manifold without boundary. Then for all $g \in C^{\infty}(M, U(l \times l ; \mathbb{C}))$ one has $(d+P) \rho\left(\mathrm{Ch}^{-}(g)\right)=0$ in $\mathscr{N}_{\epsilon}\left(\Omega_{\mathbb{T}}(M \times \mathbb{T})\right)$, in particular, $\rho\left(\mathrm{Ch}^{-}(g)\right)$ induces a homology class in $\widehat{\mathrm{H}}_{\mathbb{T}}^{-}(L M)$.

Remark 5.3. There is an even version of $\mathrm{Ch}^{-}(g)$ given as follows: If $N$ is a manifold and $d+C$ is a connection on a trivial vector bundle over $N$, then with $R_{C}$ the curvature of the connection 1-form $C$ one defines

$$
\mathrm{Ch}^{+}(C)=\left(\mathrm{Ch}_{0}^{+}(C), \mathrm{Ch}_{1}^{+}(C), \ldots\right) \in \mathscr{C}_{\epsilon}^{+}\left(\Omega_{\mathbb{T}}(N \times \mathbb{T})\right)
$$

by

$$
\mathrm{Ch}_{n}^{+}(C):=\operatorname{Tr}_{n}\left[1 \otimes\left(C-\vartheta_{\mathbb{T}} \wedge R_{C}\right)^{\otimes n}\right]
$$


which by an analogous calculation as in the proof of Theorem 5.1 is seen to satisfy

$$
(b+B) \mathrm{Ch}^{+}(C)=0 \quad \text { in } \mathscr{N}_{\epsilon}\left(\Omega_{\mathbb{T}}(N \times \mathbb{T})\right) .
$$

Then, there holds an even/odd periodicity, that is, one can obtain $\mathrm{Ch}^{-}(g)$ from its even variant by a fiber integration: indeed, by varying $s \in I$ in

$$
A^{s}(g) \in \Omega_{\mathbb{T}}(M, \operatorname{Mat}(l \times l ; \mathbb{C}))
$$

we get a form

$$
A(g) \in \Omega_{\mathbb{T}}(M \times I, \operatorname{Mat}(l \times l ; \mathbb{C}))
$$

and can consider the fibration

$$
\pi: M \times I \longrightarrow M .
$$

Then, for the connection $d+\tilde{A}_{g}$ on the trivial vector bundle over $M \times I$, where $\tilde{A}_{g}:=\pi^{*} A_{g}$, one has, using the definitions of $\mathcal{A}^{s}(g)$ and $\mathcal{B}^{s}(g)$ that

$$
\mathrm{Ch}^{-}(g)=\int_{I} \iota_{\partial_{I}} \mathrm{Ch}^{+}\left(\tilde{A}_{g}\right)=\pi_{*} \mathrm{Ch}^{+}\left(\tilde{A}_{g}\right),
$$

the integration along the fibers of $\pi$.

The odd Chern character $\operatorname{ch}^{-}(g) \in \Omega^{-}(M)$ is the closed odd differential form defined by

$$
\operatorname{ch}^{-}(g):=\operatorname{Tr}\left[\sum_{j=0}^{\infty} \frac{(-1)^{j} j !}{(2 j+1) !}\left(g^{-1} d g\right)^{\wedge(2 j+1)}\right],
$$

and the odd Bismut-Chern character is the differential form

$$
\operatorname{Bch}^{-}(g)=\left(\operatorname{Bch}_{1}^{-}(g), \operatorname{Bch}_{3}^{-}(g), \ldots\right) \in \widehat{\Omega}^{-}(L M)
$$

defined by

$$
\begin{gathered}
\operatorname{Bch}_{2 n-1}^{-}(g)=\operatorname{Tr}\left[\int_{0}^{1} \int_{\left\{0 \leq t_{1} \leq \ldots t_{n} \leq 1\right\}} \sum_{j=1}^{n} \bigwedge_{i=1}^{j-1} / /\left.\right|_{t_{i}} ^{s}(g) R_{g}^{s}\left(t_{i}\right) \bigwedge / / /_{t_{j}}^{s}(g) \dot{A}_{g}^{s}\left(t_{j}\right)\right. \\
\left.\bigwedge_{l=j+1}^{n} / / /_{t_{l}}^{s}(g) R_{g}^{s}\left(t_{l}\right) / / /_{1}^{s}(g) d t_{1} \cdots d t_{n} d s\right],
\end{gathered}
$$

where

$$
\dot{A}_{g}^{s}=\frac{d}{d s} A_{g}^{s}=\omega_{g} \in \Omega^{1}(M, \operatorname{Mat}(l \times l ; \mathbb{C})),
$$

and where $/ / . s(g)$ denotes the parallel transport with respect to the connection $d+s \omega_{g}$ on the trivial vector buncle over $M$.

Theorem 5.4. Assume $M$ is a compact Riemannian manifold, possibly with boundary, and let $g \in C^{\infty}(M, U(l \times l ; \mathbb{C}))$. Then one has $\left.\rho\left(\mathrm{Ch}^{-}(g)\right)\right|_{M}=\mathrm{ch}^{-}(g)$, and if $M$ has no boundary then $\mathrm{Bch}^{-}(g)=\rho\left(\mathrm{Ch}^{-}(g)\right)$. 
Note that in view of Corollary 5.2. Theorem 5.4 provides a new proof of

$$
(d+P) \mathrm{Bch}^{-}(g)=0
$$

We refer the reader to [18] for a variant of this result.

Proof of Theorem 5.4. The formula $\left.\rho\left(\mathrm{Ch}^{-}(g)\right)\right|_{M}=\mathrm{ch}^{-}(g)$ is a simple consequence of the definitions, once one has noticed the formula

$$
\left.\rho\left\langle\left(\alpha_{0}+\vartheta_{\mathbb{T}} \wedge \beta_{0}\right) \otimes \cdots \otimes\left(\alpha_{n}+\vartheta_{\mathbb{T}} \wedge \beta_{n}\right)\right\rangle\right|_{M}=\alpha_{0} \wedge \cdots \wedge \alpha_{n} .
$$

In order to see $\mathrm{Bch}^{-}(g)=\rho(g)$, given $t, s \in I$ define

$$
V^{s}(g, t) \in \widehat{\Omega}^{-}(L M, \operatorname{Mat}(l \times l ; \mathbb{C}))
$$

by

$$
\begin{aligned}
V_{2 n+1}^{s}(g, t)=\int_{\left\{0 \leq t_{1} \leq \ldots t_{n+1} \leq t\right\}} & \sum_{j=1}^{n+1} \bigwedge_{i=1}^{j-1} / / t_{t_{i}}^{s}(g) R_{g}^{s}\left(t_{i}\right) \bigwedge / /_{t_{j}}^{s}(g) \dot{A}_{g}^{s}\left(t_{j}\right) \\
& \times \bigwedge_{l=j+1}^{n+1} / /_{t_{l}}^{s}(g) R_{g}^{s}\left(t_{l}\right) / /_{1}^{s}(g) d t_{1} \cdots d t_{n+1},
\end{aligned}
$$

and the differential form

$$
W^{s}(g, t) \in \widehat{\Omega}^{-}(L M, \operatorname{Mat}(l \times l ; \mathbb{C}))
$$

by

$$
\begin{aligned}
& W_{2 n+1}^{s}(g, t)=\sum_{k=n+1}^{\infty} \sum_{r, j_{1}, \cdots, j_{n}=1, \text { pairwise distinct }}^{k} \\
& \times \int_{\left\{0 \leq t_{1} \leq \ldots t_{k} \leq t\right\}} \iota A_{g}^{s}\left(t_{1}\right) \cdots R_{g}^{s}\left(t_{j_{1}}\right) \cdots \dot{A}_{g}^{s}\left(t_{r}\right) \cdots R_{g}^{s}\left(t_{j_{n}}\right) \cdots \iota A_{g}^{s}\left(t_{k}\right) d t_{1} \cdots d t_{k} .
\end{aligned}
$$

Then obviously one has

$$
\operatorname{Bch}^{-}(g)=\operatorname{Tr}\left[\left.\int_{0}^{1} V^{s}(g, t)\right|_{t=1} d s\right]
$$

and it is easily checked from the definitions that

$$
\rho\left(\mathrm{Ch}^{-}(g)\right)=\operatorname{Tr}\left[\left.\int_{0}^{1} W^{s}(g, t)\right|_{t=1} d s\right] .
$$

Thus it suffices to show that $W^{s}(g, t)=V^{s}(g, t)$ for all $t, s \in I$. To see this, the essential idea is to consider for every $t, s \in I$ the even form

$$
X^{s}(g, t)=\left(X_{0}^{s}(g, t), X_{2}^{s}(g, t), \ldots\right) \in \widehat{\Omega}^{+}(L M, \operatorname{Mat}(l \times l ; \mathbb{C}))
$$


which is defined by

$$
\begin{aligned}
& X_{0}^{s}(g, t)=/{ }_{t}^{s}(g), \\
& \frac{d}{d t} X_{2 n}^{s}(g, t)=X_{2 n}^{s}(g, t) \iota A_{g}^{s}(t)+X_{2 n-2}^{s}(g, t) R_{g}^{s}(t), \\
& \left.X_{2 n}^{s}(g, t)\right|_{t=0}=0 \quad \text { for all } n \geq 1,
\end{aligned}
$$

and the odd form

$$
Y^{s}(g, t)=\left(Y_{1}^{s}(g, t), Y_{3}^{s}(g, t), \ldots\right) \in \Omega^{-}(L M, \operatorname{Mat}(l \times l ; \mathbb{C}))
$$

which is defined by

$$
\begin{aligned}
& \frac{d}{d t} Y_{1}^{s}(g, t)=Y_{1}^{s}(g, t) \iota A_{g}^{s}(t)+X_{0}^{s}(g, t) \dot{A}_{g}^{s}(t), \\
& \frac{d}{d t} Y_{2 n+1}^{s}(g, t)=Y_{2 n+1}^{s}(g, t) \iota A_{g}^{s}(t)+Y_{2 n-1}^{s}(g, t) R_{g}^{s}(t)+X_{2 n}^{s}(g, t) \dot{A}_{g}^{s}(t) \quad \forall n \geq 1, \\
& \left.Y_{2 n+1}^{s}(g, t)\right|_{t=0}=0 \quad \text { for all } n .
\end{aligned}
$$

Noting that the sum that defines $W_{2 n+1}^{s}(g, t)$ converges uniformly in $t$ so that one can interchange $d / d t$ with $\sum_{k=n+1}^{\infty}$, it is now easily checked that both $t \mapsto W^{s}(g, t)$ and $t \mapsto V^{s}(g, t)$ solve the IVP's which define $Y^{s}(g, t)$, so that

$$
V^{s}(g, t)=W^{s}(g, t)=Y^{s}(g, t) \quad \text { for all } t, s \in I,
$$

as was claimed.

Remark 5.5. If $N$ is a compact manifold without boundary and given a connection $d+C$ over a trivial vector bundle over $N$, the even Bismut-Chern character is the differential form

$$
\operatorname{Bch}^{+}(C)=\left(\operatorname{Bch}_{0}^{+}(C), \operatorname{Bch}_{2}^{+}(C), \ldots\right) \in \widehat{\Omega}^{+}(L N)
$$

defined by

$$
\mathrm{Bch}_{2 n}^{+}(C)=\operatorname{Tr}\left[\int_{\left\{0 \leq t_{1} \leq \ldots t_{n} \leq 1\right\}} \bigwedge_{i=1}^{n} / / t_{t_{i}}^{C} R_{C}\left(t_{i}\right) / /{ }_{1}^{C} d t_{1} \cdots d t_{n}\right],
$$

where $R_{C}$ is again the curvature of $d+C$ and $/ /{ }^{C}$ is the parallel transport with respect to $d+C$. Then one has another even/odd periodicity as in Remark 5.3 we can consider $A_{g}^{s}$ as defining a connection 1 -form $\tilde{A}_{g}$ over a trivial vector bundle over $M \times I$. However, since $M \times I$ is a manifold with boundary, it is convenient to embed it in a larger manifold, say

$$
\chi: M \times I \hookrightarrow M \times J
$$


where $J=(-1,2)$. Therefore, we extend $A_{g}^{s}$ to $s \in J$, consider it as defining a connection 1-form $\tilde{A}_{g}$ over a trivial vector bundle over $M \times J$.

The corresponding curvature

$$
R_{\tilde{A}_{g}} \in \Omega^{2}(M \times J, \operatorname{Mat}(l \times l ; \mathbb{C}))
$$

is given by varying $s \in J$ in

$$
R_{g}^{s}+d s \wedge \dot{A}_{g}^{s} \in \Omega^{2}(M, \operatorname{Mat}(l \times l ; \mathbb{C})) .
$$

Since $\iota_{\partial J} R_{\tilde{A}_{g}}=\dot{A}_{g}^{s}$, after restricting to loops fibering over $J$, we immediately get that under integration along the fibers of

$$
\pi: M \times I \longrightarrow M
$$

one has

$$
\operatorname{Bch}_{2 n-1}^{-}(g)=\int_{I} \chi^{*} \iota_{\partial_{J}} \operatorname{Bch}_{2 n}^{+}\left(\tilde{A}_{g}\right)=\pi_{*} \chi^{*} \operatorname{Bch}_{2 n}^{+}\left(\tilde{A}_{g}\right)
$$

\section{References}

[1] Atiyah, M. : Circular symmetry and stationary-phase approximation. Colloquium in honor of Laurent Schwartz, vol. 1, (Palaiseau, 1983). Asterisque, 1(131):43-59, 1985.

[2] Bismut, J.-M.: Index theorem and equivariant cohomology on the loop space. Comm. Math. Phys. 98 (1985), no. 2, 213-237.

[3] Bismut, J.-M.: Duistermaat-Heckman formulas and index theory. Geometric aspects of analysis and mechanics, Progr. Math., vol. 292, Birkhauser/Springer, New York, 2011, pp. 1-55.

[4] Bismut, J.-M. \& Freed, D.S.: The analysis of elliptic families. I. Metrics and connections on determinant bundles. Comm. Math. Phys., Volume 106, Number 1 (1986), 159-176.

[5] Bismut, J.-M. \& Freed, D.S.: The analysis of elliptic families. II. Dirac operators, eta invariants, and the holonomy theorem. Comm. Math. Phys., Volume 107, Number 1 (1986), 103-163.

[6] Chen, K.-T.: Iterated integrals of differential forms and loop space homology. Ann. of Math. (2) 97 (1973), 217-246.

[7] Connes, A.: Entire cyclic cohomology of Banach algebras and characters of $\theta$-summable Fredholm modules. K-Theory 1, (1988), 519-548.

[8] Getzler, E.: The odd Chern character in cyclic homology and spectral flow. Topology 32 (1993), no. 3, 489-507. 
[9] Getzler, E. The Thom class of Mathai and Quillen and probability theory. Stochastic Analysis and Applications (Lisbon 1989). Prog. Probab. 26, Birkhauser, 1991, 111-122.

[10] Getzler, E. \& Szenes, A.: On the Chern character of theta-summable Fredholm modules. J. Func. Anal. 84 (1989), 343-357.

[11] Getzler, E.; Jones, John D. S.; Petrack, Scott: Differential forms on loop spaces and the cyclic bar complex. Topology 30 (1991), no. 3, 339-371.

[12] Getzler, E.; Szenes, A.: On the Chern character of theta-summable Fredholm modules. J. Func. Anal. 84 (1989), 343-357.

[13] Hanisch, F. \& Ludewig, M.: Supersymmetric Path Integrals I: Differential Forms on the Loop Space. arXiv:1709.10027.

[14] Shen, Shu: Private communication.

[15] Simons, J. \& Sullivan, D.: Structured Vector Bundles define Differential KTheory. arXiv:0810.4935.

[16] Tradler, Thomas; Wilson, Scott O.; Zeinalian, Mahmoud: Equivariant holonomy for bundles and abelian gerbes. Comm. Math. Phys. 315 (2012), no. 1, $39-108$.

[17] Waldmann, S.: Geometric Wave Equations. arXiv:1208.4706v1.

[18] Wilson, S.O: A loop group extension of the odd Chern character. J. Geom. Phys. 102 (2016), 32-43. 